\title{
Finite dimensional compact and unitary Lie superalgebras
}

\author{
Saeid Azamª, Karl-Hermann Neeb ${ }^{\mathrm{b}}$ \\ a School of Mathematics, Institute for Research in Fundamental Sciences (IPM), P.O.Box: \\ 19395-5746, Tehran, Iran, and Department of Mathematics, University of Isfahan, P.O.Box \\ 81745-163, Isfahan, Iran. \\ b Department of Mathematics, Friedrich-Alexander University Erlangen-Nuremberg, Cauer- \\ strasse 11, 91058, Erlangen, Germany.
}

\begin{abstract}
Motivated by the theory of unitary representations of finite dimensional Lie supergroups, we describe those Lie superalgebras which have a faithful finite dimensional unitary representation. We call these Lie superalgebras unitary. This is achieved by describing the classification of real finite dimensional compact simple Lie superalgebras, and analyzing, in a rather elementary and direct way, the decomposition of reductive Lie superalgebras ( $\mathfrak{g}$ is a semisimple $\mathfrak{g}_{\overline{0}}$-module) over fields of characteristic zero into ideals.

Keywords: unitary, reductive, compact Lie superalgebras, central extension, spin representation

$M S C: 17 \mathrm{~B} 20 ; 22 \mathrm{E} 45$.
\end{abstract}

\section{Introduction}

A real Lie algebra $\mathfrak{k}$ is called compact if it is the Lie algebra of a compact Lie group ([HN12, Sect. 12.1]). There are many characterizations of compact Lie algebras:

Theorem 1.1. For a finite dimensional real Lie algebra $\mathfrak{k}$, the following are equivalent:

(i) $\mathfrak{k}$ is compact.

(ii) $\mathfrak{k}$ carries a positive definite invariant symmetric bilinear form.

(iii) $\mathfrak{k}$ has a faithful finite dimensional unitary representation, i.e., it is isomorphic to a subalgebra of $\mathfrak{u}(n, \mathbb{C})$ for some $n \in \mathbb{N}$.

${ }^{*}$ Corresponding author at: Department of Mathematics, University of Isfahan, P.O.Box 81745-163, Isfahan, Iran.

E-mail addresses: azam@sci.ui.ac.ir (S. Azam), neeb@math.fau.de (K.-H. Neeb). 
In this paper we discuss variants of compactness for finite dimensional real Lie superalgebras.

Definition 1.2. (a) A Lie superalgebra $\mathfrak{g}=\mathfrak{g}_{\overline{0}} \oplus \mathfrak{g}_{\overline{1}}$ over a field $\mathbb{K}$ of characteristic zero is called reductive if $\mathfrak{g}$ is a semisimple module for the Lie algebra $\mathfrak{g}_{\overline{0}}$ with respect to the adjoint representation. It is called semisimple if it contains no non-zero solvable ideal.

A simple reductive Lie superalgebra is called classical (cf. [Ka77). For a Lie superalgebra $\mathfrak{g}$ we always assume $\mathfrak{g}_{\overline{1}} \neq\{0\}$, unless otherwise stated.

(b) A real Lie superalgebra $\mathfrak{g}$ is called compact if $\mathfrak{g}_{\overline{0}}$ is a compactly embedded subalgebra, i.e., the subgroup of $\operatorname{Aut}(\mathfrak{g})$ generated by $e^{\operatorname{adg} \mathfrak{g}_{0}}$ has compact closure. Since this implies that $\mathfrak{g}$ is a semisimple $\mathfrak{g}_{\overline{0}}$-module, compact Lie superalgebras are reductive.

(c) A real Lie superalgebra $\mathfrak{g}$ is called unitary if it has a faithful unitary representation, i.e., if it is isomorphic to a subalgebra of some Lie superalgebra $\mathfrak{u}(p \mid q ; \mathbb{C})$.

If $\mathfrak{g}$ is a real Lie algebra, i.e., $\mathfrak{g}_{\overline{1}}=\{0\}$, then compactness and unitarity are the same by Theorem 1.1. For Lie superalgebras it is easy to see that unitarity implies compactness, but that the converse is false in general. The main goal of this paper is to describe the structure of unitary Lie superalgebras. This is in particular motivated by the theory of unitary representations of Lie supergroups (CCTV06, NS11]), for which a thorough understanding of the finite dimensional unitary Lie superalgebras is a fundamental ingredient.

In our structural analysis of unitary Lie superalgebras we proceed as follows. In Section 2 we describe the classification of compact simple Lie superalgebras (Theorem 2.3) and discuss some of their relatives. There are only 4 families:

$$
\mathfrak{s u}(n \mid m ; \mathbb{C}), n>m, \quad \mathfrak{p s u}(n \mid n ; \mathbb{C}), n \geq 2, \quad \mathfrak{p q}(n), n>2, \quad \text { and } \quad \mathfrak{c}(n), n \geq 2
$$

This classification result is derived from Parker's classification of real forms of classical Lie superalgebras ([Pa80] ) by inspecting which of these real forms are compact. In Section 3 we take a first closer look at special properties of unitary Lie superalgebras. A crucial property of a unitary Lie superalgebra is the existence of linear functionals $\omega$ on $\mathfrak{g}_{\overline{0}}$ for which the symmetric bilinear form $(X, Y) \mapsto \omega([X, Y])$ on $\mathfrak{g}_{\overline{1}}$ is positive definite. This implies in particular that the closed convex cone $\mathcal{C}(\mathfrak{g}) \subseteq \mathfrak{g}_{\overline{0}}$ generated by the elements $[X, X], X \in \mathfrak{g}_{\overline{1}}$, is pointed and that $\mathfrak{g}_{\overline{0}}$ has non-trivial center (cf. [NS11]). Although these requirements fail for some simple compact Lie superalgebras, they always possess unitary central extensions.

Section 4 provides the main information on the decomposition of reductive Lie superalgebras into ideals. Since this analysis goes through without additional problem over fields of characteristic zero, it is carried out in this context. Here we focus on the essential case where $\mathfrak{g}$ is a reductive Lie superalgebra satisfying $\mathfrak{g}_{\overline{0}}=\left[\mathfrak{g}_{\overline{1}}, \mathfrak{g}_{\overline{1}}\right]$, i.e., where $\mathfrak{g}$ is generated by its odd part $\mathfrak{g}_{\overline{1}}$. Our main result is the Structure Theorem 4.8 which describes the commutator algebra $[\mathfrak{g}, \mathfrak{g}]$ as a quotient of a direct sum of the center and of Lie superalgebras $\mathfrak{g}(j)$ and $\mathfrak{c}(k)$ by a central subspace. Here $\mathfrak{g}(j) / \mathfrak{z}(\mathfrak{g}(j))$ is a 
classical Lie superalgebra and $\mathfrak{c}(k) / \mathfrak{z}(\mathfrak{c}(k)) \cong \mathfrak{k} \otimes \Lambda_{1}$, where $\mathfrak{k}$ is a simple Lie algebra and $\Lambda_{n}$ denotes the Graßmann algebra with $n$ generators. The results in this section are closely related to Elduque's results on reductive Lie superalgebras over fields of characteristic zero ([E196]; see also [BR78]). In principle we could have derived our results from his, but our approach is rather elementary, very direct and never requires algebraic closedness of the base field. In particular it works over $\mathbb{R}$. [

In the final Section 5 everything is put together to obtain a description of the structure of unitary Lie superalgebras. Here one of the main results is Theorem 5.2 which asserts that if $\mathfrak{g}$ is unitary, then the ideals $\mathfrak{g}(j)$ are isomorphic to

$$
\mathfrak{s u}(n \mid m ; \mathbb{C}), n \geq m \geq 1, \quad \mathfrak{q}(n), n \geq 2, \quad \text { or } \quad \mathfrak{c}(n), n \geq 2 .
$$

Here the Lie superalgebras of the form $\mathfrak{s u}(n \mid m ; \mathbb{C}), n>m$, and $\mathfrak{c}(n)$ must be direct summands, whereas the Lie superalgebras $\mathfrak{q}(n)$ and $\mathfrak{s u}(n \mid n ; \mathbb{C})$ intersect the center nontrivially. The ideals $\mathfrak{c}(k)$ are direct sums of a central ideal and a one-dimensional central extension of $\mathfrak{k} \otimes \Lambda_{1}$, where $\mathfrak{k}$ is a compact simple Lie algebra. Finally, we show that all these types occur.

\section{Contents}

1 Introduction 1

2 Compact classical Lie superalgebras

3 Unitary Lie superalgebras

3.1 The spin representation . . . . . . . . . . . . . . . 8

3.2 Central extensions . . . . . . . . . . . . . . . . . . . . . 9

4 Decomposition theory 10

5 The structure of unitary Lie superalgebras 18

\section{Compact classical Lie superalgebras}

In this section we start our analysis with the classification of compact simple Lie superalgebras (Theorem 2.3). In passing, we introduce some related extensions and discuss their matrix realizations. We recall that a real Lie superalgebra $\mathfrak{g}$ is called compact if $\mathfrak{g}_{\overline{0}}$ is a compactly embedded subalgebra, i.e., the subgroup of Aut( $\left.\mathfrak{g}\right)$ generated by

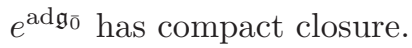

According to Ka77, the classical simple complex Lie superalgebras are grouped in 6 infinite series and 3 exceptional types.

\footnotetext{
${ }^{1}$ For an inductive classification of reductive Lie superalgebras equipped with an even non-degenerate supersymmetric $\mathfrak{g}$-invariant bilinear form see $\mathrm{Be00}$.
} 
- $\begin{cases}A(n, m) & :=\mathfrak{s l}(n+1 \mid m+1, \mathbb{C}) \text { for } n>m \geq 0 \\ A(n, n) & :=\mathfrak{p s l}(n+1 \mid n+1 ; \mathbb{C}):=\mathfrak{s l}(n+1 \mid n+1, \mathbb{C}) / \mathbb{C} \mathbf{1} \text { for } n>0 .\end{cases}$

- $B(n, m):=\mathfrak{o s p}(2 n+1 \mid 2 m), n \geq 0, m>0$.

- $C(n):=\mathfrak{o s p}(2 \mid 2 n-2), n \geq 2$.

- $D(n, m):=\mathfrak{o s p}(2 n \mid 2 m), n \geq 2, m>0$.

- $Q(n):=\widetilde{Q}(n) / \mathbb{C} 1, \widetilde{Q}(n):=\left\{\left(\begin{array}{ll}a & b \\ b & a\end{array}\right) \in \mathfrak{s l}(n+1 \mid n+1, \mathbb{C}): \operatorname{tr} b=0\right\}$, for $n \geq 2$ (the queer Lie superalgebra).

- $P(n):=\left\{\left(\begin{array}{cc}a & b \\ c & -a^{\top}\end{array}\right) \in \mathfrak{s l}(n+1 \mid n+1, \mathbb{C}): b=b^{\top}, c^{\top}=-c\right\}, n>1$.

- $G(3), F(4)$, and $D(2,1, \alpha)$, where $\alpha \in \mathbb{C} \backslash\{0,-1\}$.

Remark 2.1. The even parts of the complex classical nonexceptional Lie superalgebras are

$$
\left\{\begin{array}{l}
A(n, m)_{\overline{0}} \cong \mathfrak{s l}(n+1, \mathbb{C}) \oplus \mathfrak{s l}(m+1, \mathbb{C}) \oplus \mathbb{C} \\
A(n, n)_{\overline{0}}=\mathfrak{s l}(n+1, \mathbb{C})^{2}
\end{array}\right.
$$

$\mathfrak{o} \mathfrak{s p}(n \mid 2 m)_{\overline{0}} \cong \mathfrak{s o}(n, \mathbb{C}) \oplus \mathfrak{s p}(2 m, \mathbb{C}), \quad Q(n)_{\overline{0}} \cong \mathfrak{s l}(n+1, \mathbb{C}), \quad P(n)_{\overline{0}} \cong \mathfrak{s l}(n+1, \mathbb{C})$.

In particular, the even part has non-zero (one-dimensional) center for $A(n, m), n \neq m$ and $C(n)$.

(b) According to $\mathrm{Pa} 80$, any real form $\mathfrak{g}$ of a complex classical Lie superalgebra $\mathfrak{g}_{\mathbb{C}}$ is determined up to isomorphism by its even part $\mathfrak{g}_{\overline{0}}$.

(c) In view of [Ka77, Thm. 1, §2.3.4], the Cartan-Killing form of $A(n, n)$ and $Q(n)$ vanishes, whereas the Cartan-Killing form of $A(n, m), n \neq m$, and of $C(n)$ is nondegenerate. This further implies that all derivations of $A(n, m)$ and $C(n)$ are inner ([Ka77, Prop. 2.3.4]). Since derivations and cocycles are in one-to-one correspondence via the map

$$
\operatorname{der}(\mathfrak{g}) \rightarrow Z^{2}(\mathfrak{g}, \mathbb{C}), \quad D \mapsto \omega_{D}, \quad \omega_{D}(x, y):=\kappa(D x, y)=\operatorname{str}(\operatorname{ad}(D x) \operatorname{ad} y),
$$

where $\kappa$ is the Cartan-Killing form, and inner derivations correspond to trivial cocycles, it follows that all central extensions of $A(n, m), n \neq m$, and $C(n)$ are trivial.

Examples 2.2. (a) The prototype of a compact Lie superalgebra is the real form

$$
\mathfrak{g}:=\mathfrak{u}(p \mid q ; \mathbb{C}):=\left\{\left(\begin{array}{cc}
A & B \\
i B^{*} & D
\end{array}\right) \in \mathfrak{g l}(p \mid q ; \mathbb{C}): A \in \mathfrak{u}(p ; \mathbb{C}), D \in \mathfrak{u}(q ; \mathbb{C}), B \in M_{p, q}(\mathbb{C})\right\}
$$

of the complex Lie superalgebra $\mathfrak{g l}(p \mid q ; \mathbb{C})$. Then $\mathfrak{g}_{\overline{1}} \cong M_{p, q}(\mathbb{C})$ and the $\mathfrak{g}_{\overline{0}}$-module structure on this space is equivalent to $\mathbb{C}^{p} \otimes \overline{\mathbb{C}^{q}}$, so that $\mathfrak{g}_{\overline{1}}$ is a simple $\mathfrak{g}_{\overline{0}}$-module. 
An odd element $X \in \mathfrak{g l}(p \mid q ; \mathbb{C})$ is contained in $\mathfrak{u}(p \mid q ; \mathbb{C})$ if and only if $X^{*}=-i X$. Therefore the involution on $\mathfrak{g l}(p \mid q ; \mathbb{C})$ that leads to the real form $\mathfrak{u}(p \mid q ; \mathbb{C})$ can be written as

$$
\sigma\left(X_{0}+X_{1}\right)=-X_{0}^{*}+i X_{1}^{*} \quad \text { for } \quad X_{0} \in \mathfrak{g}_{\mathbb{C}, \overline{0}}, X_{1} \in \mathfrak{g}_{\mathbb{C}, \overline{1}} .
$$

For later use we record the following property of $\mathfrak{g}$ :

$$
[X, X]=2 X^{2}=2 i X^{*} X \neq 0 \quad \text { for } \quad 0 \neq X \in \mathfrak{g}_{\overline{1}} .
$$

(b) The Lie superalgebra $\mathfrak{g}=\mathfrak{s u}(n \mid n ; \mathbb{C}):=\{X \in \mathfrak{u}(n \mid n ; \mathbb{C})$ : $\operatorname{str} X=0\}$ has the non-zero center $\mathfrak{z}=\mathbb{R} i \mathbf{1}=\mathfrak{z}\left(\mathfrak{g}_{\overline{0}}\right)$ and $\mathfrak{g}_{\overline{0}} \cong \mathfrak{z} \oplus \mathfrak{s u}(n, \mathbb{C})^{2}$, so that $\mathfrak{z}$ acts trivially on $\mathfrak{g}_{\overline{1}}$. Since $M_{n}(\mathbb{C}) \cong \mathbb{C}^{n} \otimes \overline{\mathbb{C}^{n}}$ is a simple module of $\mathfrak{g}_{\overline{0}}$, we see that for $\mathfrak{g}$ and also for the simple Lie superalgebra $\mathfrak{p s u}(n \mid n ; \mathbb{C})=\mathfrak{g} / \mathfrak{z}(\mathfrak{g})$, the representation of $\mathfrak{g}_{\overline{0}}$ on $\mathfrak{g}_{\overline{1}}$ is irreducible.

We also note that

$$
i \mathbf{1}=X^{2}=\frac{1}{2}[X, X] \quad \text { for } \quad X:=\left(\begin{array}{cc}
0 & \mathbf{1} \\
i \mathbf{1} & 0
\end{array}\right) \in \mathfrak{g}_{\overline{1}},
$$

shows that $\mathfrak{z}=\mathfrak{z}(\mathfrak{g}) \subseteq[\mathfrak{g}, \mathfrak{g}]$, that is, $\mathfrak{g}$ is a non-trivial central extension of $\mathfrak{p} \mathfrak{s u}(n \mid n ; \mathbb{C}):=$ $\mathfrak{g} / \mathfrak{z}(\mathfrak{g})$. Since the center of the even part of this quotient is trivial, it is not unitary (cf. Lemma 3.4below); all unitary representations $\pi$ of $\mathfrak{g}$ with $\pi(i \mathbf{1})=0$ kill the element $X$, hence are trivial because the complexification $\mathfrak{p s l}(n \mid n ; \mathbb{C})$ of $\mathfrak{p s u}(n \mid n ; \mathbb{C})$ is a simple complex Lie superalgebra (see also Ja94).

For $n \neq m$, the center $\mathfrak{z}$ of $\mathfrak{s u}(n \mid m ; \mathbb{C})_{\overline{0}}$ is non-zero and acts non-trivially on $\mathfrak{s u}(n \mid m ; \mathbb{C})_{\overline{1}}$.

(c) For $n \geq 0$, the Lie superalgebra

$$
\mathfrak{q}(n):=\widetilde{Q}(n) \cap \mathfrak{s u}(n+1 \mid n+1 ; \mathbb{C})=\left\{\left(\begin{array}{ll}
a & b \\
b & a
\end{array}\right): a^{*}=-a, b^{*}=-i b, \operatorname{tr} b=0\right\},
$$

is a compact real form of $\widetilde{Q}(n)$. Accordingly, $\mathfrak{p q}(n):=\mathfrak{q}(n) / \mathbb{R} i \mathbf{1}$ is a compact real form of the Lie superalgebra $Q(n)$, which is simple for $n \geq 1$. Note that

$$
\left\{b \in M_{n+1}(\mathbb{C}): b^{*}=-i b\right\}=(1-i) \mathfrak{u}(n+1, \mathbb{C})
$$

shows that the module structure for $\mathfrak{q}(n)_{\overline{0}} \cong \mathfrak{u}(n+1, \mathbb{C})$ on $\mathfrak{q}(n)_{\overline{1}}$ is equivalent to the adjoint representation on $\mathfrak{s u}(n+1, \mathbb{C})$, hence irreducible.

We also note that $\mathfrak{z}\left(\mathfrak{q}(n)_{\overline{0}}\right)=\{0\}$, that $\mathfrak{q}(n)=[\mathfrak{q}(n), \mathfrak{q}(n)]$ and that it embeds naturally as a hyperplane ideal into

$$
\widehat{\mathfrak{q}}(n):=\left\{\left(\begin{array}{ll}
a & b \\
b & a
\end{array}\right) \in \mathfrak{g l}(n+1 \mid n+1 ; \mathbb{C}): a^{*}=-a, b^{*}=-i b\right\} .
$$

Therefore $D:=\operatorname{ad}\left(\begin{array}{cc}0 & \mathbf{1} \\ i \mathbf{1} & 0\end{array}\right)$ defines an exterior odd derivation of $\mathfrak{q}(n)$ with $D^{2}=0$. 
(d) Let $\mathfrak{c}(n)$ be the compact real form of $C(n)=\mathfrak{o s p}(2 \mid 2 n-2), n \geq 2$, with

$$
\mathfrak{c}(n)_{\overline{0}} \cong \mathfrak{s o}(2, \mathbb{R}) \oplus \mathfrak{s p}(n-1) \cong \mathbb{R} \oplus \mathfrak{u}(n-1 ; \mathbb{H})
$$

and

$$
\mathfrak{c}(n)_{\overline{1}} \cong \mathbb{H}^{n-1} \quad \text { with } \quad\left(\mathfrak{c}(n)_{\overline{1}}\right)_{\mathbb{C}} \cong M_{2}(\mathbb{C})^{n-1} \cong \mathbb{C}^{2} \otimes \mathbb{C}^{2(n-1)} .
$$

As $\mathbb{H}^{n-1}$ is an irreducible $\mathfrak{s p}(n-1)$-module, the action of $\mathfrak{c}(n)_{\overline{0}}$ on $\mathfrak{c}(n)_{\overline{1}}$ is irreducible.

We also note that $\mathfrak{z}\left(\mathfrak{c}(n)_{\overline{0}}\right) \cong \mathfrak{s o}(2, \mathbb{R}) \neq\{0\}$. The Cartan-Killing form of $\mathfrak{c}(n)$ is non-zero, hence non-degenerate, and this implies that all derivations are inner and that all central extensions are trivial (cf. [Ka77, Prop. 2.3.4]).

Theorem 2.3. (Compact classical Lie superalgebras) Every compact simple real Lie superalgebra $\mathfrak{g}$ is isomorphic to one of the following:

(a) $\mathfrak{s u}(n \mid m ; \mathbb{C})$ for $n>m \geq 1$.

(b) $\mathfrak{p s u}(n \mid n ; \mathbb{C})$ for $n \geq 2$.

(c) $\mathfrak{p q}(n)=\mathfrak{q}(n) / i \mathbb{R} \mathbf{1}, n \geq 2$.

(d) $\mathfrak{c}(n)$, the compact real form of $C(n)=\mathfrak{o s p}(2 \mid 2 n-2), n \geq 2$.

For all these simple real Lie superalgebras $\mathfrak{g}$, the $\mathfrak{g}_{\overline{0}}$-module $\mathfrak{g}_{\overline{1}}$ is irreducible, $\mathfrak{z}\left(\mathfrak{g}_{\overline{0}}\right)=\{0\}$ for $\mathfrak{p s u}(n \mid n ; \mathbb{C})$ and $\mathfrak{p q}(n)$, and $\mathfrak{z}\left(\mathfrak{g}_{0}\right) \cong \mathbb{R}$ for $\mathfrak{s u}(n \mid m ; \mathbb{C}), n>m$ and $\mathfrak{c}(n)$.

Proof. The compactness of $\mathfrak{g}$ implies that it is classical. If $\mathfrak{g}_{\mathbb{C}}$ is not simple, then $\mathfrak{g}$ is a complex simple Lie superalgebra, considered as real one. Then ad $\mathfrak{g}_{\overline{0}}$ is a complex subalgebra of $\operatorname{der}(\mathfrak{g})$ and this can only generate a relatively compact group of automorphisms if it is trivial, i.e., $\mathfrak{g}_{\overline{0}} \subseteq \mathfrak{z}(\mathfrak{g})$, but this contradicts the simplicity of $\mathfrak{g}$.

Therefore $\mathfrak{g}_{\mathbb{C}}$ is simple and $\mathfrak{g}$ is a compact real form of the classical Lie superalgebra $\mathfrak{g}_{\mathbb{C}}$. From the classification of the real forms in [Pa80, Thm. 2.5] it follows that compact real forms are unique whenever they exist. We also see that the exceptional algebras and the algebras $P(n)$ have no compact real forms because the Lie algebras $\mathfrak{s l}(2, \mathbb{R})$, $\mathfrak{s o}(p, q), p q>1$, and $\mathfrak{s u}^{*}(n), n>0$, are not compact. Further, the algebras $B(n, m)$, $m>0$, and $D(n, m)$ have no compact real form because the Lie algebras $\mathfrak{s o}^{*}(2 n)$, $n>12$ are never compact.

For $A(n, m), Q(n)$ and $C(n)$, the compact real forms are given by (a), (b), (c) and (d) respectively. The simplicity of the $\mathfrak{g}_{\overline{0}}$-module $\mathfrak{g}_{\overline{1}}$ follows from the discussion in Examples 2.2, which also implies the remaining assertion on $\mathfrak{z}\left(\mathfrak{g}_{\overline{0}}\right)$.

Remark 2.4. In F13, Fioresi constructs compact real forms in terms of the root decomposition. Fixing a Cartan subalgebra and a Chevalley basis $\left\{H_{j}, X_{\alpha}\right\}$ (see FG12, Chapter 3]), she thus obtains for any classical Lie superalgebra satisfying $\left[X_{\alpha}, X_{\alpha}\right]=0$ for all $\alpha \in \Delta$ a compact real form.

\footnotetext{
${ }^{2} \operatorname{In} \mathfrak{s o *}(2 n)=\mathfrak{s o}(2 n, \mathbb{C}) \cap \mathfrak{s l}(n, \mathbb{H})$ the subalgebra $\mathfrak{u}(n, \mathbb{C})$ is maximally compact and equality implies $n=1$.
} 


\section{$3 \quad$ Unitary Lie superalgebras}

In this section we take a closer look at the class of unitary Lie superalgebras and some of their specific properties.

Definition 3.1. A (finite dimensional) unitary representation for a finite dimensional Lie superalgebra $\mathfrak{g}$ is a pair $(\mathcal{H}, \rho)$ where $\mathcal{H}=\mathcal{H}_{\overline{0}} \oplus \mathcal{H}_{\overline{1}}$ is a finite dimensional complex super Hilbert space with the corresponding sesquilinear positive definite even hermitian form $\langle\cdot, \cdot\rangle: \mathcal{H} \times \mathcal{H} \rightarrow \mathbb{C}$, and $\rho: \mathfrak{g} \rightarrow \operatorname{End}_{\mathbb{C}}(\mathcal{H})$ is a (real) Lie superalgebra homomorphism satisfying

$$
\langle\rho(X) v, w\rangle=\left\langle v,-i^{|X|} \rho(X) w\right\rangle,
$$

for $X \in \mathfrak{g}$ and $v, w \in \mathcal{H}$. If we write

$$
\mathfrak{u}\left(\mathcal{H}_{\overline{0}} \mid \mathcal{H}_{\overline{1}}\right):=\left\{\left(\begin{array}{cc}
a & b \\
i b^{*} & d
\end{array}\right) \in \mathfrak{g l}(\mathcal{H}): a \in \mathfrak{u}\left(\mathcal{H}_{\overline{0}}\right), d \in \mathfrak{u}\left(\mathcal{H}_{\overline{1}}\right), b \in \operatorname{Hom}\left(\mathcal{H}_{\overline{1}}, \mathcal{H}_{\overline{0}}\right)\right\},
$$

then unitary representations correspond to homomorphism of Lie superalgebras $\rho: \mathfrak{g} \rightarrow \mathfrak{u}\left(\mathcal{H}_{\overline{0}} \mid \mathcal{H}_{\overline{1}}\right)$.

Since we can form direct sums of unitary representations, we have the following trivial lemma:

Lemma 3.2. For a finite dimensional Lie superalgebra, the following are equivalent:

(i) The finite dimensional unitary representations of $\mathfrak{g}$ separate the points.

(ii) $\mathfrak{g}$ has a faithful finite dimensional unitary representation.

(iii) $\mathfrak{g}$ is isomorphic to a subalgebra of some $\mathfrak{u}(p \mid q ; \mathbb{C})$.

Definition 3.3. (a) We call a finite dimensional real Lie superalgebra $\mathfrak{g}$ unitary if it has a faithful finite dimensional unitary representation.

(b) For a finite dimensional Lie superalgebra $\mathfrak{g}$, we write $\mathcal{C}(\mathfrak{g}) \subseteq \mathfrak{g}_{\overline{0}}$ for the closed convex cone generated by the elements of the form $[X, X], X \in \mathfrak{g}_{\overline{1}}$.

Lemma 3.4. If $\mathfrak{g}$ is unitary then the following assertions hold:

(i) $\mathfrak{g}$ is compact, i.e., the even part $\mathfrak{g}_{\overline{0}}$ is compactly embedded in $\mathfrak{g}$.

(ii) $[X, X] \neq 0$ for $0 \neq X \in \mathfrak{g}_{\overline{1}}$.

(iii) $\mathcal{C}(\mathfrak{g})$ is pointed, i.e., $\mathcal{C}(\mathfrak{g}) \cap-\mathcal{C}(\mathfrak{g})=\{0\}$.

(iv) There exists a $\mathfrak{g}_{0}$-invariant linear functional $\omega \in \mathfrak{g}_{0}^{*}$, for which the form

$$
\kappa_{\omega}: \mathfrak{g}_{\overline{1}} \times \mathfrak{g}_{\overline{1}} \rightarrow \mathbb{R}, \quad \kappa_{\omega}\left(X_{1}, Y_{1}\right):=\omega\left(\left[X_{1}, Y_{1}\right]\right)
$$

is positive definite. 
(v) $\mathfrak{z}\left(\mathfrak{g}_{\overline{0}}\right) \neq\{0\}$.

Proof. (i)-(iii) We may assume that $\mathfrak{g} \subseteq \mathfrak{h}:=\mathfrak{u}(p \mid q ; \mathbb{C})$. Then (i) follows from the fact that $\mathfrak{h}_{\overline{0}}$ is compactly embedded in $\mathfrak{h}$, and (ii) from (2.1). Further (2.1) implies that

$$
\mathcal{C}(\mathfrak{g}) \subseteq \mathfrak{C}(\mathfrak{h}) \subseteq\{X \in \mathfrak{u}(p, \mathbb{C}) \oplus \mathfrak{u}(q, \mathbb{C}): \operatorname{Spec}(-i X) \geq 0\},
$$

which implies that $\mathcal{C}(\mathfrak{g})$ is pointed.

(iv) Since the cone $\mathcal{C}(\mathfrak{g})$ is pointed, its dual cone $\mathcal{C}(\mathfrak{g})^{\star} \subseteq \mathfrak{g}_{0}^{*}$ has interior points. Since the group $K$ generated by $e^{\text {adgo }}$ has compact closure by $(\mathrm{i}), \operatorname{Int}\left(\mathfrak{C}(\mathfrak{g})^{\star}\right)$ contains a fixed point $\omega$ for this group (cf. [NS11, Lemma 5.1.2]). Then $\omega(Y)>0$ for any non-zero element $Y \in \mathfrak{C}(\mathfrak{g})$, and therefore (ii), (iii) imply that $\kappa_{\omega}$ is positive definite on $\mathfrak{g}_{\overline{1}}$. Further, the invariance of $\omega$ under $K$, and hence under $\mathfrak{g}_{\overline{0}}$, implies that $\kappa_{\omega}$ is $\mathfrak{g}_{0}$-invariant.

(v) Since $\omega$ is $\mathfrak{g}_{\overline{0}}$-invariant, it vanishes on $\left[\mathfrak{g}_{\overline{0}}, \mathfrak{g}_{\overline{0}}\right]$, so that $\mathfrak{g}_{\overline{0}}=\mathfrak{z}\left(\mathfrak{g}_{\overline{0}}\right) \oplus\left[\mathfrak{g}_{\overline{0}}, \mathfrak{g}_{\overline{0}}\right]$ implies that the center is non-zero.

Combining Lemma 3.4(v) with Theorem 2.3. we obtain immediately:

Proposition 3.5. The simple compact Lie superalgebras $\mathfrak{p s u}(n \mid n ; \mathbb{C}), n>0$, and $\mathfrak{p q}(n)$, $n \geq 2$, are not unitary.

Remark 3.6. (a) More examples of compact Lie superalgebras which are not unitary can be obtained as follows. A Lie superalgebra $\mathfrak{g}$ is called a Clifford-Heisenberg Lie superalgebra if $\mathfrak{g}_{\overline{0}} \subseteq \mathfrak{z}(\mathfrak{g})$. Then the bracket is completely determined by the symmetric bilinear form

$$
\beta: \mathfrak{g}_{\overline{1}} \times \mathfrak{g}_{\overline{1}} \rightarrow \mathfrak{g}_{\overline{0}}, \quad(X, Y) \mapsto[X, Y] .
$$

If $\operatorname{dim} \mathfrak{g}_{\overline{0}}=1$ and $\beta$ is indefinite, then $\mathfrak{C}(\mathfrak{g})=\mathfrak{g}_{0}$ is not pointed, so that $\mathfrak{g}$ is not unitary.

In view of Lemma 3.4, unitarity even requires that $\beta$ is positive or negative definite. The spin representation associated to a positive definite form $\beta$ implies that $\mathfrak{g}$ is unitary if $\beta$ is positive definite (cf. Subsection 3.1)

(b) If $\mathfrak{g}$ is a compact Lie superalgebra, then $\mathfrak{g}_{0}$ is in particular a compact Lie algebra ( HN12, Prop. 12.1.4]). Hence the ideal $\left[\mathfrak{g}_{\overline{1}}, \mathfrak{g}_{\overline{1}}\right]$ of $\mathfrak{g}_{\overline{0}}$ has a complement $\mathfrak{c}$, and

$$
\mathfrak{g} \cong\left(\left[\mathfrak{g}_{\overline{1}}, \mathfrak{g}_{\overline{1}}\right] \oplus \mathfrak{g}_{\overline{1}}\right) \rtimes \mathfrak{c} \quad \text { with } \quad\left[\mathfrak{c},\left[\mathfrak{g}_{\overline{1}}, \mathfrak{g}_{\overline{1}}\right]\right]=\{0\} .
$$

If, conversely, $\mathfrak{c}$ is a compact Lie algebra and $\mathfrak{h}$ a compact Lie superalgebra on which $\mathfrak{c}$ acts by even derivations $\alpha: \mathfrak{c} \rightarrow \operatorname{der}(\mathfrak{h})_{\overline{0}}$ with $\alpha(\mathfrak{c})\left(\mathfrak{h}_{\overline{0}}\right)=\{0\}$ and $e^{\alpha(\mathfrak{c})_{\overline{1}}} \subseteq \operatorname{GL}\left(\mathfrak{h}_{\overline{1}}\right)$ relatively compact, then $\mathfrak{h} \rtimes \mathfrak{c}$ is a compact Lie superalgebra. Therefore, for most issues concerning the structure theory of compact Lie superalgebras, it suffices to assume that $\mathfrak{g}$ is generated by $\mathfrak{g}_{\overline{1}}$.

\subsection{The spin representation}

Let $V$ and $\mathcal{H}$ be finite dimensional complex Hilbert spaces. A representation of the canonical anticommutation relations $(C A R)$ is an antilinear map $a: V \rightarrow B(\mathcal{H})$ satisfying

$$
a(f) a(g)+a(g) a(f)=0 \quad \text { and } \quad a(f) a(g)^{*}+a(g)^{*} a(f)=\langle g, f\rangle \mathbf{1} \quad \text { for } \quad f, g \in V .
$$


For every complex Hilbert space $V$, we obtain a representation of the CAR on the exterior algebra $\Lambda(V)=\bigoplus_{k=0}^{\infty} \Lambda^{k}(V)$, endowed with the natural scalar product, by

$$
a_{0}(f)^{*}\left(f_{1} \wedge \cdots \wedge f_{n}\right)=f \wedge f_{1} \wedge \cdots \wedge f_{n}
$$

and

$$
a_{0}(f)\left(f_{1} \wedge \cdots \wedge f_{n}\right)=\sum_{j=1}^{n}(-1)^{j+1}\left\langle f_{j}, f\right\rangle f_{1} \wedge \cdots \wedge \widehat{f}_{j} \wedge \cdots \wedge f_{n}
$$

(cf. Ot95).

The complex Clifford-Heisenberg Lie superalgebra associated to the complex Hilbert space $V$ is the Lie superalgebra

$$
\mathfrak{c h}(V):=\mathbb{C} \oplus V \oplus \bar{V}, \quad\left[(z, v, w),\left(z^{\prime}, v^{\prime}, w^{\prime}\right)\right]:=\left(\left\langle v, w^{\prime}\right\rangle+\left\langle v^{\prime}, w\right\rangle, 0,0\right) .
$$

Any representation of the CAR leads to a complex linear representation of $\mathfrak{c h}(V)$ by

$$
\rho(z, v, w):=z \mathbf{1}+a(v)^{*}+a(w) .
$$

Accordingly, we obtain a real form of $\mathfrak{c h}(V)$ by

$$
\mathfrak{h}:=\{(z i, v,-i v): z \in \mathbb{R}, v \in V\}
$$

and any representation of the CAR defines a unitary representation of $\mathfrak{h}$. For $(0, v,-i v) \in$ $\mathfrak{h}$, we have

$$
[(0, v,-i v),(0, v,-i v)]=(\langle v,-i v\rangle+\langle v,-i v\rangle, 0,0)=\left(2 i\|v\|^{2}, 0,0\right) .
$$

We now consider the Lie superalgebra

$$
\mathfrak{g}:=\mathfrak{h} \rtimes_{D} \mathbb{R}, \quad D(i z, v,-i v):=(0, i v, v), \quad z \in \mathbb{R}, v \in V .
$$

Then $d:=(0,1) \in \mathfrak{z}\left(\mathfrak{g}_{\overline{0}}\right)$ acts non-trivially on $\mathfrak{g}_{\overline{1}}$. The Fock representation of the CAR extends to $\mathfrak{g}$ by

$$
\rho(d)\left(f_{1} \wedge \cdots \wedge f_{n}\right):=i n f_{1} \wedge \cdots \wedge f_{n} .
$$

We thus obtain for $\operatorname{dim} V=n$ a unitary irreducible representation of $\mathfrak{g}$ satisfying $\operatorname{Spec}(-i \rho(d))=\{0, \ldots, n\}$. For a detailed study of spin representations see [Va04.

\subsection{Central extensions}

In this subsection we discuss some aspects of central extensions of real Lie superalgebras that will be needed in Section 5 below.

Lemma 3.7. If $\beta: \mathfrak{g}_{\overline{1}} \times \mathfrak{g}_{\overline{1}} \rightarrow \mathbb{R}$ is a $\mathfrak{g}_{\overline{0}}$-invariant symmetric bilinear form, i.e.,

$$
\beta([X, Y], Z)+\beta(Y,[X, Z])=0 \quad \text { for } \quad X \in \mathfrak{g}_{\overline{0}}, Y, Z \in \mathfrak{g}_{\overline{1}},
$$

then

$$
\omega\left(X_{0}+X_{1}, Y_{0}+Y_{1}\right):=\beta\left(X_{1}, Y_{1}\right)
$$


is a Lie superalgebra 2-cocycle, i.e.,

$$
\widehat{\mathfrak{g}}:=\mathbb{R} \oplus \mathfrak{g} \quad \text { with } \quad\left[(z, X),\left(z^{\prime}, X^{\prime}\right)\right]:=\left(\omega\left(X, X^{\prime}\right),\left[X, X^{\prime}\right]\right)
$$

is a Lie superalgebra with $\widehat{\mathfrak{g}}_{\overline{0}}=\mathbb{R} \oplus \mathfrak{g}_{\overline{0}}$ and $\widehat{\mathfrak{g}}_{\overline{1}}=\mathfrak{g}_{\overline{1}}$.

Proof. We have to check for homogeneous elements $X, Y, Z \in \mathfrak{g}$ the relation

$$
\omega(X,[Y, Z])=\omega([X, Y], Z)+(-1)^{|X| \cdot|Y|} \omega(Y,[X, Z]) .
$$

Here the only non-trivial case is the situation where one argument, say $X$, is even and the others are odd. Then

$$
\omega(X,[Y, Z])=0=\beta([X, Y], Z)+\beta(Y,[X, Z])=\omega([X, Y], Z)+\omega(Y,[X, Z])
$$

follows from the invariance of $\beta$ under $\mathfrak{g}_{\overline{0}}$.

Proposition 3.8. If $\beta$ is a positive definite $\mathfrak{g}_{\overline{0}}$-invariant form on $\mathfrak{g}_{\overline{1}}$ and $\widehat{\mathfrak{g}}$ is the central extension defined by the cocycle (3.2), then the cone $\mathrm{C}(\widehat{\mathfrak{g}})$ is pointed.

Proof. The convex cone $\mathcal{C}(\widehat{\mathfrak{g}})$ is generated by the elements $(\beta(X, X),[X, X]), X \in \mathfrak{g}_{\overline{1}}$, and if $\beta$ is positive definite, it suffices to consider elements with $\beta(X, X)=1$. This leads to the compact subset

$$
C:=\left\{(1,[X, X]): X \in \mathfrak{g}_{\overline{1}}, \beta(X, X)=1\right\} \subseteq \mathbb{R} \times \mathfrak{g}_{\overline{0}}
$$

whose convex hull does not contain 0 . Therefore it generates a pointed convex cone.

Corollary 3.9. Every compact Lie superalgebra $\mathfrak{g}$ has a compact central extension $\widehat{\mathfrak{g}}$ for which the cone $\mathcal{C}(\widehat{\mathfrak{g}})$ is pointed.

Proof. Since $e^{\text {adg }} \mathfrak{g}_{\overline{0}}$ has compact closure, $\mathfrak{g}_{\overline{1}}$ carries a positive definite $\mathfrak{g}_{\overline{0}}$-invariant symmetric bilinear form $\beta$. Then the corresponding centrally extended Lie superalgebra $\widehat{\mathfrak{g}}$ has a pointed cone $\mathcal{C}(\widehat{\mathfrak{g}})$ by Proposition 3.8

\section{Decomposition theory}

In this section we turn to the structure of a reductive Lie superalgebra $\mathfrak{g}$ over a field $\mathbb{K}$ of characteristic zero. Since the results in this section will be applied to unitary (and so compact) Lie superalgebras, and in this case $\mathfrak{g}$ is always a semidirect sum of an ideal of $\mathfrak{g}_{\overline{0}}$ and the ideal of $\mathfrak{g}$ generated by $\mathfrak{g}_{\overline{1}}$ (cf. Remark $3.6(b)$ ), we assume in the following that

$$
\mathfrak{g}_{\overline{0}}=\left[\mathfrak{g}_{\overline{1}}, \mathfrak{g}_{\overline{1}}\right] .
$$

The main goal of this section is to decompose $\mathfrak{g}$ into center and ideals which are central extensions of simple Lie superalgebras or of algebras of the form $\mathfrak{k} \otimes \Lambda_{1}$, where $\mathfrak{k}$ is a simple Lie superalgebra. 
Let

$$
\mathfrak{b}:=\mathfrak{z}_{\mathfrak{g}_{\overline{1}}}\left(\mathfrak{g}_{\overline{0}}\right)=\left\{Y \in \mathfrak{g}_{\overline{1}} \mid\left[Y, \mathfrak{g}_{\overline{0}}\right]=\{0\}\right\} .
$$

We note that $\left[[\mathfrak{b}, \mathfrak{b}], \mathfrak{g}_{\overline{1}}\right] \subseteq\left[\mathfrak{b}, \mathfrak{g}_{\overline{0}}\right]=\{0\}$, and since $\mathfrak{g}$ is generated by $\mathfrak{g}_{\overline{1}}$, this leads to

$$
[\mathfrak{b}, \mathfrak{b}] \subseteq \mathfrak{z}(\mathfrak{g}) .
$$

Since $\mathfrak{b}$ is $\mathfrak{g}_{0}$-invariant and $\mathfrak{g}_{\overline{1}}$ is a semisimple $\mathfrak{g}_{0}$-module, we have a $\mathfrak{g}_{0}$-invariant decomposition $\mathfrak{g}_{\overline{1}}=\mathfrak{b} \oplus \mathfrak{a}$ with $\mathfrak{a}=\bigoplus_{j \in J} \mathfrak{a}_{j}$, where the $\mathfrak{a}_{j}$ are simple $\mathfrak{g}_{\overline{0}}$-submodules of $\mathfrak{g}_{\overline{1}}$. We then have

$$
\left[\left[\mathfrak{a}_{i}, \mathfrak{a}_{j}\right], \mathfrak{a}_{k}\right] \subseteq \mathfrak{a}_{k} \cap\left(\mathfrak{a}_{i}+\mathfrak{a}_{j}\right)=\{0\} \quad \text { for } \quad k \notin\{i, j\},
$$

and thus in particular

$$
\left[\left[\mathfrak{a}_{i}, \mathfrak{a}_{i}\right], \mathfrak{a}_{k}\right]=\{0\} \quad \text { for } \quad k \neq i .
$$

We likewise obtain

$$
\left[\left[\mathfrak{b}, \mathfrak{a}_{j}\right], \mathfrak{a}_{k}\right] \subseteq \mathfrak{a}_{k} \cap\left(\mathfrak{b}+\mathfrak{a}_{j}\right)=\{0\} \quad \text { for } \quad k \neq j .
$$

Note that

$$
\mathfrak{g}_{\overline{0}}=\left[\mathfrak{g}_{\overline{1}}, \mathfrak{g}_{\overline{1}}\right]=[\mathfrak{b}, \mathfrak{b}]+[\mathfrak{b}, \mathfrak{a}]+[\mathfrak{a}, \mathfrak{a}]=\underbrace{[\mathfrak{b}, \mathfrak{b}]}_{\subseteq \mathfrak{z}(\mathfrak{g})}+\sum_{j}\left[\mathfrak{b}, \mathfrak{a}_{j}\right]+\sum_{j, k}\left[\mathfrak{a}_{j}, \mathfrak{a}_{k}\right] .
$$

Since the spaces $\left[\left[\mathfrak{b}, \mathfrak{a}_{j}\right], \mathfrak{a}_{i}\right]$ and $\left[\left[\mathfrak{a}_{j}, \mathfrak{a}_{k}\right], \mathfrak{a}_{i}\right]$ are $\mathfrak{g}_{0}$-submodules of $\mathfrak{a}_{i}$, they are either zero or equal to $\mathfrak{a}_{i}$. As $[\mathfrak{b}, \mathfrak{b}]$ is central by (4.2), the relations $\mathfrak{a}_{i}=\left[\mathfrak{g}_{\overline{0}}, \mathfrak{a}_{i}\right]$, (4.3), (4.5) and (4.6) imply that

$$
\left[\left[\mathfrak{b}, \mathfrak{a}_{i}\right], \mathfrak{a}_{i}\right]=\mathfrak{a}_{i} \quad \text { or } \quad\left[\left[\mathfrak{a}_{j}, \mathfrak{a}_{i}\right], \mathfrak{a}_{i}\right]=\mathfrak{a}_{i} \quad \text { for some } j \in J .
$$

We put

$$
J_{s}:=\left\{k \in J:\left[[\mathfrak{a}, \mathfrak{a}], \mathfrak{a}_{k}\right]=\mathfrak{a}_{k}\right\}=\left\{k \in J:\left(\exists k^{\prime} \in J\right)\left[\left[\mathfrak{a}_{k}, \mathfrak{a}_{k^{\prime}}\right], \mathfrak{a}_{k}\right]=\mathfrak{a}_{k}\right\} .
$$

Let $J_{a}:=J \backslash J_{s}$ and consider $k \in J_{a}$. Then $\left[[\mathfrak{a}, \mathfrak{a}], \mathfrak{a}_{k}\right]=\{0\}$ by definition and so $\left[[\mathfrak{b}, \mathfrak{a}], \mathfrak{a}_{k}\right]=\left[\left[\mathfrak{b}, \mathfrak{a}_{k}\right], \mathfrak{a}_{k}\right]=\mathfrak{a}_{k}$ follows from (4.5). Therefore (4.7) leads to

$$
J_{a}=\left\{k \in J:\left[\left[\mathfrak{b}, \mathfrak{a}_{k}\right], \mathfrak{a}_{k}\right]=\mathfrak{a}_{k} \text { and }(\forall j \in J)\left[\left[\mathfrak{a}_{k}, \mathfrak{a}_{j}\right], \mathfrak{a}_{k}\right]=\{0\}\right\}
$$

Lemma 4.1. For each $k \in J_{s}$, there exists a unique $k^{\prime} \in J$ such that $\mathfrak{a}_{k}=\left[\left[\mathfrak{a}_{k}, \mathfrak{a}_{k^{\prime}}\right], \mathfrak{a}_{k}\right]$. Then $k^{\prime} \in J_{s}$ and $\mathfrak{a}_{k^{\prime}}=\left[\left[\mathfrak{a}_{k^{\prime}}, \mathfrak{a}_{k}\right], \mathfrak{a}_{k^{\prime}}\right]$.

Proof. Let $k \in J_{s}$ and $k^{\prime} \in J$ such that $\mathfrak{a}_{k}=\left[\left[\mathfrak{a}_{k}, \mathfrak{a}_{k^{\prime}}\right], \mathfrak{a}_{k}\right]$.

Step 1: First we show that $k^{\prime} \in J_{s}$. If $k^{\prime} \in J_{a}$, then $k \neq k^{\prime}$, so that

$$
\left[\mathfrak{a}_{k}, \mathfrak{a}_{k^{\prime}}\right]=\left[\mathfrak{a}_{k},\left[\left[\mathfrak{b}, \mathfrak{a}_{k^{\prime}}\right], \mathfrak{a}_{k^{\prime}}\right]\right] \subseteq\left[\left[\mathfrak{a}_{k}, \mathfrak{a}_{k^{\prime}}\right],\left[\mathfrak{b}, \mathfrak{a}_{k^{\prime}}\right]\right]+[\underbrace{4.5}_{=0 \text { by }},\left[\mathfrak{a}_{k},\left[\mathfrak{b}, \mathfrak{a}_{k^{\prime}}\right]\right], \mathfrak{a}_{k^{\prime}}] \subseteq\left[\mathfrak{b}, \mathfrak{a}_{k^{\prime}}\right] .
$$


With (4.5), this leads to the contradiction $\mathfrak{a}_{k}=\left[\left[\mathfrak{a}_{k}, \mathfrak{a}_{k^{\prime}}\right], \mathfrak{a}_{k}\right] \subseteq\left[\left[\mathfrak{b}, \mathfrak{a}_{k^{\prime}}\right], \mathfrak{a}_{k}\right]=\{0\}$.

Step 2: Next we show that $\mathfrak{a}_{k^{\prime}}=\left[\mathfrak{a}_{k^{\prime}},\left[\mathfrak{a}_{k}, \mathfrak{a}_{k^{\prime}}\right]\right]$. We may assume that $k \neq k^{\prime}$. As $k^{\prime} \in J_{s}$ by Step 1 , there exists a $t \in J$ such that $\mathfrak{a}_{k^{\prime}}=\left[\left[\mathfrak{a}_{k^{\prime}}, \mathfrak{a}_{t}\right], \mathfrak{a}_{k^{\prime}}\right]$. If $t=k^{\prime}$, then

$$
\left[\mathfrak{a}_{k}, \mathfrak{a}_{k^{\prime}}\right]=\left[\mathfrak{a}_{k},\left[\left[\mathfrak{a}_{k^{\prime}}, \mathfrak{a}_{k^{\prime}}\right], \mathfrak{a}_{k^{\prime}}\right]\right] \subseteq[\underbrace{\left[\mathfrak{a}_{k},\left[\mathfrak{a}_{k^{\prime}}, \mathfrak{a}_{k^{\prime}}\right]\right]}_{=0}, \mathfrak{a}_{k^{\prime}}]+\left[\left[\mathfrak{a}_{k}, \mathfrak{a}_{k^{\prime}}\right],\left[\mathfrak{a}_{k^{\prime}}, \mathfrak{a}_{k^{\prime}}\right]\right] \subseteq\left[\mathfrak{a}_{k^{\prime}}, \mathfrak{a}_{k^{\prime}}\right]
$$

leads with (4.4) to the contradiction

$$
\mathfrak{a}_{k}=\left[\left[\mathfrak{a}_{k}, \mathfrak{a}_{k^{\prime}}\right], \mathfrak{a}_{k}\right] \subseteq\left[\left[\mathfrak{a}_{k^{\prime}}, \mathfrak{a}_{k^{\prime}}\right], \mathfrak{a}_{k}\right]=\{0\} .
$$

Thus $t \neq k^{\prime}$. If $t \neq k$, then $t, k, k^{\prime}$ are distinct and so

$$
\left[\mathfrak{a}_{k}, \mathfrak{a}_{k^{\prime}}\right]=\left[\mathfrak{a}_{k},\left[\left[\mathfrak{a}_{k^{\prime}}, \mathfrak{a}_{t}\right], \mathfrak{a}_{k^{\prime}}\right]\right] \subseteq[\underbrace{\left[\mathfrak{a}_{k},\left[\mathfrak{a}_{k^{\prime}}, \mathfrak{a}_{t}\right]\right]}_{=0}, \mathfrak{a}_{k^{\prime}}]+\left[\left[\mathfrak{a}_{k}, \mathfrak{a}_{k^{\prime}}\right],\left[\mathfrak{a}_{k^{\prime}}, \mathfrak{a}_{t}\right]\right] \subseteq\left[\mathfrak{a}_{k^{\prime}}, \mathfrak{a}_{t}\right]
$$

leads to the contradiction $\mathfrak{a}_{k}=\left[\left[\mathfrak{a}_{k}, \mathfrak{a}_{k^{\prime}}\right], \mathfrak{a}_{k}\right] \subseteq\left[\left[\mathfrak{a}_{t}, \mathfrak{a}_{k^{\prime}}\right], \mathfrak{a}_{k}\right]=\{0\}$. We conclude that $t=k$.

Step 3: Now we show the uniqueness of $k^{\prime}$. Suppose that $s \neq t \in J$ satisfy

$$
\mathfrak{a}_{k}=\left[\mathfrak{a}_{k},\left[\mathfrak{a}_{k}, \mathfrak{a}_{s}\right]\right]=\left[\mathfrak{a}_{k},\left[\mathfrak{a}_{k}, \mathfrak{a}_{t}\right]\right] .
$$

By the symmetry proved in the previous step,

$$
\mathfrak{a}_{s}=\left[\mathfrak{a}_{s},\left[\mathfrak{a}_{s}, \mathfrak{a}_{k}\right]\right] \quad \text { and } \quad \mathfrak{a}_{t}=\left[\mathfrak{a}_{t},\left[\mathfrak{a}_{t}, \mathfrak{a}_{k}\right]\right]
$$

This leads to

$$
\begin{aligned}
{\left[\mathfrak{a}_{k}, \mathfrak{a}_{s}\right] } & =\left[\left[\mathfrak{a}_{k},\left[\mathfrak{a}_{t}, \mathfrak{a}_{k}\right]\right], \mathfrak{a}_{s}\right] \subseteq\left[\left[\mathfrak{a}_{k}, \mathfrak{a}_{s}\right],\left[\mathfrak{a}_{t}, \mathfrak{a}_{k}\right]\right]+\left[\left[\left[\mathfrak{a}_{t}, \mathfrak{a}_{k}\right], \mathfrak{a}_{s}\right], \mathfrak{a}_{k}\right] \\
& \subseteq\left[\mathfrak{a}_{t}, \mathfrak{a}_{k}\right]+\left[\left[\left[\mathfrak{a}_{s}, \mathfrak{a}_{t}\right], \mathfrak{a}_{k}\right], \mathfrak{a}_{k}\right]+\left[\left[\left[\mathfrak{a}_{s}, \mathfrak{a}_{k}\right], \mathfrak{a}_{t}\right], \mathfrak{a}_{k}\right] \\
& \subseteq\left[\mathfrak{a}_{t}, \mathfrak{a}_{k}\right]+\left[\mathfrak{a}_{k}, \mathfrak{a}_{k}\right] .
\end{aligned}
$$

Similarly,

$$
\left[\mathfrak{a}_{k}, \mathfrak{a}_{t}\right] \subseteq\left[\mathfrak{a}_{s}, \mathfrak{a}_{k}\right]+\left[\mathfrak{a}_{k}, \mathfrak{a}_{k}\right]
$$

If $k \notin\{s, t\}$, then (4.3) and (4.4) lead to the contradiction

$$
\mathfrak{a}_{s}=\left[\mathfrak{a}_{s},\left[\mathfrak{a}_{s}, \mathfrak{a}_{k}\right]\right] \subseteq\left[\mathfrak{a}_{s},\left[\mathfrak{a}_{t}, \mathfrak{a}_{k}\right]\right]+\left[\mathfrak{a}_{s},\left[\mathfrak{a}_{k}, \mathfrak{a}_{k}\right]\right]=\{0\} .
$$

If $k=s$, then $s \neq t$ and (4.9) yield

$$
\mathfrak{a}_{t}=\left[\mathfrak{a}_{t},\left[\mathfrak{a}_{t}, \mathfrak{a}_{k}\right]\right] \subseteq\left[\mathfrak{a}_{t},\left[\mathfrak{a}_{s}, \mathfrak{a}_{k}\right]\right]+\left[\mathfrak{a}_{t},\left[\mathfrak{a}_{k}, \mathfrak{a}_{k}\right]\right]=\{0\},
$$

which is absurd. By symmetry, a similar argument works for the case $k=t$. This shows that $t=s$ and the proof is complete.

Lemma 4.2. $\left[\left[\mathfrak{a}_{k}, \mathfrak{a}_{k}\right], \mathfrak{a}_{k}\right]=\{0\}$ if and only if $\left[\mathfrak{a}_{k}, \mathfrak{a}_{k}\right] \subseteq \mathfrak{z}(\mathfrak{g})$. This holds in particular for $k \in J_{a}$ and $k \in J_{s}$ with $k \neq k^{\prime}$. 
Proof. Clearly $\left[\mathfrak{a}_{k}, \mathfrak{a}_{k}\right] \subseteq \mathfrak{z}(\mathfrak{g})$ implies $\left[\left[\mathfrak{a}_{k}, \mathfrak{a}_{k}\right], \mathfrak{a}_{k}\right]=\{0\}$. If, conversely, $\left[\left[\mathfrak{a}_{k}, \mathfrak{a}_{k}\right], \mathfrak{a}_{k}\right]=$ $\{0\}$, then (4.4) leads to

$$
\left[\left[\mathfrak{a}_{k}, \mathfrak{a}_{k}\right], \mathfrak{g}_{\overline{1}}\right]=\left[\left[\mathfrak{a}_{k}, \mathfrak{a}_{k}\right], \mathfrak{a}\right]=\left[\left[\mathfrak{a}_{k}, \mathfrak{a}_{k}\right], \mathfrak{a}_{k}\right]=\{0\},
$$

so that $\left[\mathfrak{a}_{k}, \mathfrak{a}_{k}\right] \subseteq \mathfrak{z}(\mathfrak{g})$ follows from $\mathfrak{g}_{\overline{0}}=\left[\mathfrak{g}_{\overline{1}}, \mathfrak{g}_{\overline{1}}\right]$.

If $k \in J_{a}$, then $\left[\left[\mathfrak{a}_{k}, \mathfrak{a}_{k}\right], \mathfrak{a}_{k}\right]=\{0\}$ by definition. If $k \in J_{s}$ and $k \neq k^{\prime}$, then the uniqueness of $k^{\prime}$ implies that $\left[\left[\mathfrak{a}_{k}, \mathfrak{a}_{k}\right], \mathfrak{a}_{k}\right]=\{0\}$.

Lemma 4.3. (i) If $k \in J_{s}$, then $\left[\mathfrak{b}, \mathfrak{a}_{k}\right] \subseteq\left[\mathfrak{a}_{k}, \mathfrak{a}_{k^{\prime}}\right] \cap\left[\mathfrak{g}_{\overline{0}}, \mathfrak{g}_{\overline{0}}\right]$.

(ii) $\left[\mathfrak{a}_{k}, \mathfrak{a}_{s}\right]=\{0\}$ if $k \in J_{a}$ and $s \neq k$, or $k \in J_{s}$ and $s \notin\left\{k, k^{\prime}\right\}$.

(iii) If $j, k \in J_{s}$ and $k \notin\left\{j, j^{\prime}\right\}$, then $\left[\mathfrak{a}_{k}, \mathfrak{a}_{k^{\prime}}\right] \cap\left[\mathfrak{a}_{j}, \mathfrak{a}_{j^{\prime}}\right] \subseteq \mathfrak{z}(\mathfrak{g})$.

Proof. (i) follows from

$$
\left[\mathfrak{b}, \mathfrak{a}_{k}\right]=\left[\mathfrak{b},\left[\mathfrak{a}_{k},\left[\mathfrak{a}_{k}, \mathfrak{a}_{k^{\prime}}\right]\right]\right] \subseteq\left[\left[\mathfrak{b}, \mathfrak{a}_{k}\right],\left[\mathfrak{a}_{k}, \mathfrak{a}_{k^{\prime}}\right]\right]+[\mathfrak{a}_{k}, \underbrace{\left.\left[\mathfrak{b},\left[\mathfrak{a}_{k}, \mathfrak{a}_{k^{\prime}}\right]\right]\right]}_{=0} \subseteq\left[\mathfrak{a}_{k}, \mathfrak{a}_{k^{\prime}}\right] \cap\left[\mathfrak{g}_{\overline{0}}, \mathfrak{g}_{\overline{0}}\right] .
$$

(ii) Assume first that $k \in J_{s}$ and $s \notin\left\{k, k^{\prime}\right\}$. Then

$$
\begin{aligned}
{\left[\mathfrak{a}_{s}, \mathfrak{a}_{k}\right] } & =\left[\mathfrak{a}_{s},\left[\mathfrak{a}_{k},\left[\mathfrak{a}_{k}, \mathfrak{a}_{k^{\prime}}\right]\right]\right] \subseteq\left[\left[\mathfrak{a}_{s}, \mathfrak{a}_{k}\right],\left[\mathfrak{a}_{k}, \mathfrak{a}_{k^{\prime}}\right]\right]+[\underbrace{\left[\mathfrak{a}_{s},\left[\mathfrak{a}_{k}, \mathfrak{a}_{k^{\prime}}\right]\right.}_{=0}, \mathfrak{a}_{k}] \\
& \subseteq\left[\mathfrak{a}_{k}, \mathfrak{a}_{k^{\prime}}\right] \cap\left[\mathfrak{g}_{\overline{0}}, \mathfrak{g}_{\overline{0}}\right] .
\end{aligned}
$$

If $s \in J_{s}$, then $s \notin\left\{k, k^{\prime}\right\}$ implies $k \notin\left\{s, s^{\prime}\right\}$ and we get similarly

$$
\left[\mathfrak{a}_{s}, \mathfrak{a}_{k}\right] \subseteq\left[\mathfrak{a}_{s}, \mathfrak{a}_{s^{\prime}}\right] \cap\left[\mathfrak{g}_{\overline{0}}, \mathfrak{g}_{\overline{0}}\right]
$$

Thus $\left[\mathfrak{a}_{s}, \mathfrak{a}_{k}\right] \subseteq\left[\mathfrak{a}_{s}, \mathfrak{a}_{s^{\prime}}\right] \cap\left[\mathfrak{a}_{k}, \mathfrak{a}_{k^{\prime}}\right] \cap\left[\mathfrak{g}_{\overline{0}}, \mathfrak{g}_{\overline{0}}\right]$, and (4.4) thus leads to $\left[\left[\mathfrak{a}_{s}, \mathfrak{a}_{k}\right], \mathfrak{a}_{\overline{1}}\right]=\{0\}$, which in turn shows that

$$
\left[\mathfrak{a}_{s}, \mathfrak{a}_{k}\right] \subseteq\left[\mathfrak{g}_{\overline{0}}, \mathfrak{g}_{\overline{0}}\right] \cap \mathfrak{z}(\mathfrak{g}) \subseteq\left[\mathfrak{g}_{\overline{0}}, \mathfrak{g}_{\overline{0}}\right] \cap \mathfrak{z}\left(\mathfrak{g}_{\overline{0}}\right)=\{0\}
$$

Here we use that $\mathfrak{g}_{\overline{0}}$ is reductive.

If $s \in J_{a}$, then

$$
\begin{aligned}
{\left[\mathfrak{a}_{k}, \mathfrak{a}_{s}\right] } & =\left[\mathfrak{a}_{k},\left[\mathfrak{a}_{s},\left[\mathfrak{a}_{s}, \mathfrak{b}\right]\right]\right] \subseteq\left[\left[\mathfrak{a}_{k}, \mathfrak{a}_{s}\right],\left[\mathfrak{a}_{s}, \mathfrak{b}\right]\right]+\underbrace{\left[\left[\mathfrak{a}_{k},\left[\mathfrak{a}_{s}, \mathfrak{b}\right]\right.\right.}_{=0 \text { by }}], \mathfrak{a}_{s}] \\
& =\left[\left[\mathfrak{a}_{k}, \mathfrak{a}_{s}\right],\left[\mathfrak{a}_{s}, \mathfrak{b}\right]\right] \subseteq[\underbrace{\left[\left[\mathfrak{a}_{k}, \mathfrak{a}_{s}\right], \mathfrak{a}_{s}\right]}_{=0 \text { by } 4[.8}, \mathfrak{b}]+[\underbrace{\left[\left[\mathfrak{a}_{k}, \mathfrak{a}_{s}\right], \mathfrak{b}\right]}_{=0}, \mathfrak{a}_{s}]=\{0\} .
\end{aligned}
$$

The case $k \in J_{a}$ and $s \in J_{s}$ follows from the first part.

Finally assume that $k, s \in J_{a}, k \neq s$. Then

$$
\left[\mathfrak{a}_{k}, \mathfrak{a}_{s}\right]=\left[\mathfrak{a}_{k},\left[\mathfrak{a}_{s},\left[\mathfrak{a}_{s}, \mathfrak{b}\right]\right]\right] \subseteq\left[\left[\mathfrak{a}_{k}, \mathfrak{a}_{s}\right],\left[\mathfrak{a}_{s}, \mathfrak{b}\right]\right]+[\mathfrak{a}_{s}, \underbrace{\left[\mathfrak{a}_{k},\left[\mathfrak{a}_{s}, \mathfrak{b}\right]\right]}_{=0 \text { by }}] \subseteq\left[\mathfrak{a}_{s}, \mathfrak{b}\right] \cap\left[\mathfrak{g}_{\overline{0}}, \mathfrak{g}_{\overline{0}}\right] .
$$


We likewise get $\left[\mathfrak{a}_{k}, \mathfrak{a}_{s}\right] \subseteq\left[\mathfrak{a}_{k}, \mathfrak{b}\right]$, so that (4.5) leads to $\left[\mathfrak{a}_{k}, \mathfrak{a}_{s}\right] \subseteq \mathfrak{z}(\mathfrak{g})$, and thus to $\left[\mathfrak{a}_{k}, \mathfrak{a}_{s}\right] \subseteq \mathfrak{z}\left(\mathfrak{g}_{\overline{0}}\right) \cap\left[\mathfrak{g}_{\overline{0}}, \mathfrak{g}_{\overline{0}}\right]=\{0\}$.

(iii) Let $\mathfrak{z}:=\left[\mathfrak{a}_{k}, \mathfrak{a}_{k^{\prime}}\right] \cap\left[\mathfrak{a}_{j}, \mathfrak{a}_{j^{\prime}}\right]$. Then (4.4) shows that

$$
\left[\mathfrak{z}, \mathfrak{g}_{1}\right]=\left[\mathfrak{z}, \mathfrak{a}_{k}+\mathfrak{a}_{k^{\prime}}\right] \subseteq\left[\left[\mathfrak{a}_{i}, \mathfrak{a}_{j^{\prime}}\right], \mathfrak{a}_{k}+\mathfrak{a}_{k^{\prime}}\right]=\{0\},
$$

so that the assertion follows from $\mathfrak{g}_{\overline{0}}=\left[\mathfrak{g}_{\overline{1}}, \mathfrak{g}_{\overline{1}}\right]$.

Remark 4.4. If $\mathfrak{m} \subseteq \mathfrak{g}_{\overline{1}}$ is a $\mathfrak{g}_{0}$-invariant subspace, then $\mathfrak{m}+[\mathfrak{m}, \mathfrak{m}] \subseteq \mathfrak{g}$ is a $\mathfrak{g}_{0}$-invariant subalgebra of $\mathfrak{g}$. In particular, [ $\mathfrak{m}, \mathfrak{m}] \unlhd \mathfrak{g}_{\overline{0}}$ is an ideal.

We now consider the following subalgebras of $\mathfrak{g}$ (cf. Remark 4.4):

$$
\begin{aligned}
& \mathfrak{g}(k):=\mathfrak{a}_{k}+\mathfrak{a}_{k^{\prime}}+\left[\mathfrak{a}_{k}, \mathfrak{a}_{k}\right]+\left[\mathfrak{a}_{k}, \mathfrak{a}_{k^{\prime}}\right]+\left[\mathfrak{a}_{k^{\prime}}, \mathfrak{a}_{k^{\prime}}\right] \quad \text { for } \quad k \in J_{s}, \\
& \mathfrak{c}(k):=\mathfrak{a}_{k}+\left[\mathfrak{a}_{k}, \mathfrak{a}_{k}\right]+\left[\mathfrak{b}, \mathfrak{a}_{k}\right] \quad \text { for } \quad k \in J_{a} .
\end{aligned}
$$

For $k \neq k^{\prime}$ in $J_{s}$, the relation $\left[\mathfrak{a}_{k}, \mathfrak{a}_{k}\right]+\left[\mathfrak{a}_{k^{\prime}}, \mathfrak{a}_{k^{\prime}}\right] \subseteq \mathfrak{z}(\mathfrak{g})$ (Lemma 4.3) implies that

$$
\mathfrak{g}(k) \cap\left[\mathfrak{g}_{\overline{0}}, \mathfrak{g}_{\overline{0}}\right] \subseteq\left[\mathfrak{a}_{k}, \mathfrak{a}_{k^{\prime}}\right] .
$$

From Lemma 4.3(i), we obtain

$$
[\mathfrak{b}, \mathfrak{a}]=\sum_{k \in J_{a}}\left[\mathfrak{b}, \mathfrak{a}_{k}\right]+\sum_{k \in J_{s}}\left[\mathfrak{b}, \mathfrak{a}_{k}\right] \subseteq \sum_{k \in J_{a}}\left[\mathfrak{b}, \mathfrak{a}_{k}\right]+\sum_{k \in J_{s}}\left[\mathfrak{a}_{k}, \mathfrak{a}_{k^{\prime}}\right]
$$

By Lemmas 4.2 and 4.3 (ii) we have

$$
[\mathfrak{a}, \mathfrak{a}]=\sum_{k \in J_{a}}\left[\mathfrak{a}_{k}, \mathfrak{a}_{k}\right]+\sum_{k^{\prime} \neq k \in J_{s}}\left[\mathfrak{a}_{k}, \mathfrak{a}_{k}\right]+\sum_{k \in J_{s}}\left[\mathfrak{a}_{k}, \mathfrak{a}_{k^{\prime}}\right] \subseteq \mathfrak{z}(\mathfrak{g})+\sum_{k \in J_{s}}\left[\mathfrak{a}_{k}, \mathfrak{a}_{k^{\prime}}\right] .
$$

Thus

$$
\mathfrak{g}=\underbrace{[\mathfrak{b}, \mathfrak{b}]+[\mathfrak{b}, \mathfrak{a}]+[\mathfrak{a}, \mathfrak{a}]}_{\mathfrak{g}_{\overline{0}}}+\underbrace{\mathfrak{b}+\mathfrak{a}}_{\mathfrak{g}_{\overline{1}}}=\mathfrak{b}+[\mathfrak{b}, \mathfrak{b}]+\sum_{k \in J_{a}} \mathfrak{c}(k)+\sum_{k \in J_{s}} \mathfrak{g}(k) .
$$

The structure of subalgebras $\mathfrak{g}(k)$ and $\mathfrak{c}(j), k \in J_{s}, j \in J_{a}$ and possibilities for the index sets $J_{s}$ and $J_{a}$ will be discussed in what follows, see for example Proposition 4.5 (iv), Example 4.6. Remark 4.9 and Example 5.3

Proposition 4.5. For every $j \in J_{s}$, the Lie superalgebra $\mathfrak{g}(j)$ is an ideal of $\mathfrak{g}$ with the following properties:

(i) $\mathfrak{a}_{j}$ and $\mathfrak{a}_{j^{\prime}}$ are simple $\mathfrak{g}(j)_{\overline{0}}$-modules. In particular, $\mathfrak{g}(j)_{\overline{1}}$ is a semisimple $\mathfrak{g}(j)_{\overline{0}}$ module.

(ii) $\mathfrak{z}_{\mathfrak{g}(j)_{\overline{1}}}\left(\mathfrak{g}(j)_{\overline{0}}\right)=\{0\}, \mathfrak{z}(\mathfrak{g}(j)) \subseteq \mathfrak{z}(\mathfrak{g})_{\overline{0}}$ and $\mathfrak{z}\left(\mathfrak{g}(j)_{\overline{0}}\right) \subseteq \mathfrak{z}\left(\mathfrak{g}_{\overline{0}}\right)$.

(iii) $\mathfrak{p g}(j):=\mathfrak{g}(j) / \mathfrak{z}(\mathfrak{g}(j))$ is a classical Lie superalgebra.

(iv) If $\mathbb{K}=\mathbb{R}$ and $\mathfrak{g}(j)$ is compact, then $j=j^{\prime}$. This holds in particular for every $j \in J_{s}$ if $\mathfrak{g}$ is compact. 
Proof. First we observe that $\left[\mathfrak{b}, \mathfrak{a}_{j}+\mathfrak{a}_{j^{\prime}}\right] \subseteq\left[\mathfrak{a}_{j}, \mathfrak{a}_{j^{\prime}}\right]$ by Lemma 4.3)(i). For $k \notin\left\{j, j^{\prime}\right\}$ we get $\left[\mathfrak{a}_{k}, \mathfrak{a}_{j}+\mathfrak{a}_{j^{\prime}}\right]=\{0\}$ from Lemma 4.3 (ii). This leads to

$$
\left[\mathfrak{g}_{\overline{1}}, \mathfrak{a}_{j}+\mathfrak{a}_{j^{\prime}}\right]=\left[\mathfrak{b}+\mathfrak{a}_{j}+\mathfrak{a}_{j^{\prime}}, \mathfrak{a}_{j}+\mathfrak{a}_{j^{\prime}}\right] \subseteq \mathfrak{g}(j) .
$$

As $\mathfrak{g}_{\overline{0}}=\left[\mathfrak{g}_{\overline{1}}, \mathfrak{g}_{\overline{1}}\right]$ and $\mathfrak{g}(j)$ is $\mathfrak{g}_{\overline{0}}$-invariant, this implies that $\mathfrak{g}(j) \unlhd \mathfrak{g}$.

(i) Since all subspaces $\mathfrak{a}_{k}, k \notin\left\{j, j^{\prime}\right\}$, commute with $\mathfrak{g}(j)$ by Lemma 4.3(ii) and all subspaces $\left[\mathfrak{b}, \mathfrak{a}_{k}\right], k \notin\left\{j, j^{\prime}\right\}$, commute with $\mathfrak{g}(j)$ by (4.5), Lemma 4.3(i) leads to

$$
\mathfrak{g}_{\overline{0}} \subseteq \mathfrak{z}_{\mathfrak{g}_{\overline{0}}}(\mathfrak{g}(j))+\mathfrak{g}(j)_{\overline{0}}+\left[\mathfrak{b}, \mathfrak{a}_{j}+\mathfrak{a}_{j^{\prime}}\right] \subseteq \mathfrak{z}_{\mathfrak{g}_{\overline{0}}}(\mathfrak{g}(j))+\mathfrak{g}(j)_{\overline{0}} .
$$

Therefore $\mathfrak{a}_{j}$ and $\mathfrak{a}_{j^{\prime}}$ are simple $\mathfrak{g}(j)_{\overline{0}}$-modules.

(ii) From the non-triviality of the simple $\mathfrak{g}(j)_{\overline{0}}$-modules $\mathfrak{a}_{j}$ and $\mathfrak{a}_{j^{\prime}}$, it follows that the centralizer of $\mathfrak{g}(j)_{\overline{0}}$ in $\mathfrak{g}(j)_{\overline{1}}$ vanishes. In particular, $\mathfrak{z}:=\mathfrak{z}(\mathfrak{g}(j)) \subseteq \mathfrak{g}(j)_{\overline{0}}$.

From Lemma 4.3 it follows that $\left[\mathfrak{z}, \mathfrak{g}_{\overline{1}}\right]=[\mathfrak{z}, \mathfrak{a}]=\left[\mathfrak{z}, \mathfrak{a}_{j}+\mathfrak{a}_{j^{\prime}}\right]=\{0\}$, so that $\mathfrak{z} \subseteq \mathfrak{z}(\mathfrak{g})$ because $\mathfrak{g}_{\overline{1}}$ generates $\mathfrak{g}$.

As $\mathfrak{z}\left(\mathfrak{g}(j)_{\overline{0}}\right) \unlhd \mathfrak{g}(j)_{\overline{0}}$ is $\mathfrak{g}_{\overline{0}}$-invariant, it is an abelian ideal of the reductive Lie algebra $\mathfrak{g}_{\overline{0}}$, hence central in $\mathfrak{g}_{\overline{0}}$.

(iii) Let $\mathfrak{h}:=\mathfrak{g}(j)$ and $\{0\} \neq I \unlhd \mathfrak{h}$ be an ideal. If $I_{\overline{1}}=\{0\}$, then $I=I_{\overline{0}} \subseteq \mathfrak{z}(\mathfrak{h})$.

Suppose that $I_{\overline{1}} \neq\{0\}$. If $I_{\overline{0}} \subseteq \mathfrak{z}(\mathfrak{h})$, then $\left[I_{\overline{1}}, \mathfrak{h}_{\overline{1}}\right] \subseteq I_{\overline{0}} \subseteq \mathfrak{z}(\mathfrak{h})$, which leads to $\left[I_{\overline{1}}, \mathfrak{h}_{\overline{0}}\right]=\left[I_{\overline{1}},\left[\mathfrak{h}_{\overline{1}}, \mathfrak{h}_{\overline{1}}\right]\right]=\{0\}$. In view of (ii), this contradicts $I_{\overline{1}} \neq\{0\}$. We conclude that $I_{\overline{0}} \nsubseteq \mathfrak{z}(\mathfrak{h})$. This implies that either $\left[I_{\overline{0}}, \mathfrak{a}_{j}\right] \neq\{0\}$ or $\left[I_{\overline{0}}, \mathfrak{a}_{j^{\prime}}\right] \neq\{0\}$. By symmetry, we may assume $\left[I_{\overline{0}}, \mathfrak{a}_{j}\right] \neq\{0\}$. Then $\{0\} \neq\left[I_{\overline{0}}, \mathfrak{a}_{j}\right] \subseteq I_{\overline{1}} \cap \mathfrak{a}_{j}$. Since the latter is a

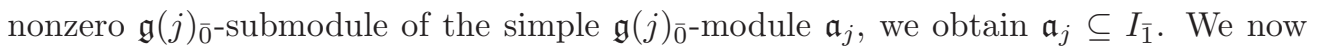
get

$$
\mathfrak{a}_{j^{\prime}}=\left[\mathfrak{a}_{j^{\prime}},\left[\mathfrak{a}_{j^{\prime}}, \mathfrak{a}_{j}\right]\right] \subseteq\left[\mathfrak{a}_{j^{\prime}},\left[\mathfrak{a}_{j^{\prime}}, I_{\overline{1}}\right]\right] \subseteq I_{\overline{1}},
$$

and thus $I=\mathfrak{h}$. This proves (iii).

(iv) Since $\mathfrak{g}(j)$ is compact, the same holds for the quotient Lie superalgebra $\mathfrak{p g}(j)$ which is classical by (iii). Hence the assertion follows from Theorem 2.3

Example 4.6. (Tangent superalgebras) Let $\mathfrak{k}$ be a simple Lie algebra. Then the tangent algebra $T \mathfrak{k} \cong \mathfrak{k} \otimes \Lambda_{1}, \Lambda_{1}=\mathbb{K}[\xi], \xi^{2}=0$, carries the structure of a Lie superalgebra, where $(T \mathfrak{k})_{\overline{0}}=\mathfrak{k} \otimes 1$ and $(T \mathfrak{k})_{\overline{1}}=\mathfrak{k} \otimes \xi$. Note that $\mathfrak{k} \otimes \xi$ is an abelian ideal. Here the brackets of odd elements vanish. Since $T \mathfrak{k} \cong \mathfrak{k}^{2}$ as a module of $\mathfrak{k} \cong(T \mathfrak{k})_{\overline{0}}$, this Lie algebra is reductive. It has an odd derivation

$$
D=\frac{\partial}{\partial \xi}: T \mathfrak{k} \rightarrow T \mathfrak{k}, \quad D(x \otimes 1+y \otimes \xi)=y \otimes 1 .
$$

Now

$$
\widehat{T} \mathfrak{k}:=T \mathfrak{k} \rtimes_{D} \mathbb{R}
$$

also is a reductive Lie superalgebra and $(\widehat{T} \mathfrak{k})_{\overline{1}} \cong \mathfrak{k} \oplus \mathbb{R}$ contains a subspace commuting with $\mathfrak{k}$ which is not central.

In the terminology from above, we then have $\mathfrak{b}=\mathbb{R}(0,1), \mathfrak{a}=\mathfrak{k} \otimes \xi$, and $J=J_{a}$ is a one-element set because $[\mathfrak{a}, \mathfrak{a}]=\{0\}$. 
Proposition 4.7. Let $k \in J_{a}$. Then $\mathfrak{c}(k) \unlhd \mathfrak{g}$ is an ideal with the following properties:

(i) $\mathfrak{k}_{k}:=\left[\mathfrak{b}, \mathfrak{a}_{k}\right]$ is a simple Lie algebra.

(ii) $\mathfrak{c}(k) / \mathfrak{z}(\mathfrak{c}(k)) \cong T \mathfrak{k}_{k}$.

(iii) $\mathfrak{c}(k)$ contains the nilpotent ideal $\mathfrak{a}_{k}+\left[\mathfrak{a}_{k}, \mathfrak{a}_{k}\right]$, hence is not semisimple.

(iv) Let $b \in \mathfrak{b}$ with $\left[b, \mathfrak{a}_{k}\right] \neq\{0\}$. Then $\widehat{\mathfrak{c}}(k):=\mathfrak{c}(k)+\mathbb{K} b+\mathbb{K}[b, b]$ is a subalgebra of $\mathfrak{g}$ for which $\widehat{\mathfrak{c}}(k) / \mathfrak{z}(\widehat{\mathfrak{c}}(k)) \cong \widehat{T}_{k}$ is semisimple.

Proof. (i), (ii) Lemma 4.3(ii) implies that $\left[\mathfrak{a}_{k}, \mathfrak{a}_{j}\right]=\{0\}$ for $j \neq k$. By (4.2) and (4.5), the centralizer of $\mathfrak{a}_{k}$ in $\mathfrak{g}_{\overline{0}}$ contains

$$
[\mathfrak{b}, \mathfrak{b}], \quad[\mathfrak{a}, \mathfrak{a}], \quad\left[\mathfrak{b}, \mathfrak{a}_{j}\right], j \neq k .
$$

We conclude that $\mathfrak{g}_{\overline{0}}=\mathfrak{z}_{\mathfrak{g}_{\overline{0}}}\left(\mathfrak{a}_{k}\right)+\left[\mathfrak{b}, \mathfrak{a}_{k}\right]$, so that $\mathfrak{a}_{k}$ is also a simple module of the ideal $\mathfrak{k}:=\left[\mathfrak{b}, \mathfrak{a}_{k}\right]$ of the reductive Lie algebra $\mathfrak{g}_{0}$ and that $\mathfrak{k}$ has an ideal complement acting trivially on $\mathfrak{a}_{k}$.

For every $b \in \mathfrak{b}$, the operator ad $b: \mathfrak{a}_{k} \rightarrow \mathfrak{k}$ is $\mathfrak{k}$-equivariant. Therefore the adjoint representation of $\mathfrak{k}$ is a sum of simple modules isomorphic to $\mathfrak{a}_{k}$, hence semisimple and a direct sum of submodules isomorphic to $\mathfrak{a}_{k}$. In particular, $\mathfrak{k}$ is semisimple, hence a direct sum of simple ideals $\mathfrak{k}_{1}, \ldots, \mathfrak{k}_{n}$. Then the ideals $\mathfrak{k}_{j}$ are simple $\mathfrak{k}$-modules with respect to the adjoint action and for $i \neq j$ they are non-isomorphic because $\mathfrak{k}_{i}$ acts trivially on $\mathfrak{k}_{j}$ and vice versa. This implies that $n=1$ and that $\mathfrak{k}$ is a simple Lie algebra. Using Lemma 4.2(ii), we see that $\mathfrak{c}(k)$ is a central extension of the Lie superalgebra

$$
\mathfrak{c}(k) / \mathfrak{z}(\mathfrak{c}(k)) \cong \mathfrak{k}+\mathfrak{a}_{k}
$$

which is isomorphic to the Lie superalgebra $T \mathfrak{k}$ from Example 4.6.

Note that $\mathfrak{k}$ annihilates a complement of $\mathfrak{a}_{k}$ in $\mathfrak{g}_{\overline{1}},\left[\mathfrak{a}_{k}, \mathfrak{a}_{k}\right]$ is central, and $\left[\mathfrak{a}_{k}, \mathfrak{g}_{\overline{1}}\right]=$ $\left[\mathfrak{a}_{k}, \mathfrak{a}_{k}\right]+\left[\mathfrak{a}_{k}, \mathfrak{b}\right]$ by Lemma 4.3(ii). We conclude that

$$
\left[\mathfrak{c}(k), \mathfrak{g}_{\overline{1}}\right]=\left[\mathfrak{a}_{k}, \mathfrak{g}_{\overline{1}}\right]+\left[\mathfrak{k}, \mathfrak{g}_{\overline{1}}\right] \subseteq \mathfrak{c}(k),
$$

and therefore $\mathfrak{c}(k) \unlhd \mathfrak{g}$ is an ideal.

(iii) follows from $\left[\mathfrak{a}_{k}, \mathfrak{a}_{k}\right] \subseteq \mathfrak{z}(\mathfrak{g})$ (Lemma 4.2).

(iv) Let $\mathfrak{k}:=\mathfrak{k}_{k}$. Since $\mathfrak{c}(k)$ is an ideal of $\mathfrak{g}$, the subspace $\widehat{\mathfrak{c}}(k)$ is a subalgebra. Now it follows from Lemma 4.2, (4.2) and (i) that $\mathfrak{z}(\widehat{\mathfrak{c}}(k))=\left[\mathfrak{a}_{k}, \mathfrak{a}_{k}\right]+\mathbb{K}[b, b]$ and $\widehat{\mathfrak{c}}(k) / \mathfrak{z}(\widehat{\mathfrak{c}}(k)) \cong T \mathfrak{k} \rtimes_{D} \mathfrak{k}$ for a derivation mapping $D \in \operatorname{der}(T \mathfrak{k})$ with $D^{2}=0$ mapping $\mathfrak{a}_{k}$ in a $\mathfrak{k}$-equivariant fashion to $\mathfrak{k}$. This implies that it is isomorphic to $\widehat{T} \mathfrak{k}$. To see that $\widehat{T} \mathfrak{k}$ is semisimple, let $\mathfrak{m} \unlhd \widehat{T} \mathfrak{k}$ be a solvable ideal. Then $\mathfrak{m}_{\overline{0}}$ must be an abelian ideal of $(\widehat{T} \mathfrak{k})_{\overline{0}} \cong \mathfrak{k}$, hence trivial. Therefore $\mathfrak{m} \subseteq(\widehat{T} \mathfrak{k})_{\overline{1}}$ and the ideal property implies $[b, \mathfrak{m}]=\{0\}$, which leads to $\mathfrak{m} \subseteq \mathbb{K} b$. As $\left[b, \mathfrak{a}_{k}\right] \neq\{0\}$, we obtain $\mathfrak{m}=\{0\}$.

In any Lie superalgebra $\mathfrak{g}$ we have $[\mathfrak{g}, \mathfrak{g}]_{\overline{1}}=\left[\mathfrak{g}_{\overline{0}}, \mathfrak{g}_{\overline{1}}\right]$. If, in addition, $\mathfrak{g}$ is reductive, then the $\mathfrak{g}_{\overline{0}}$-module $\mathfrak{g}_{\overline{1}}$ is semisimple, so that $\mathfrak{z}(\mathfrak{g})_{\overline{1}}$ intersects $[\mathfrak{g}, \mathfrak{g}]$ trivially. Therefore $\mathfrak{z}(\mathfrak{g})_{\overline{1}}$ is a direct summand and it does no harm to assume that $\mathfrak{z}(\mathfrak{g}) \subseteq \mathfrak{g}_{\overline{1}}$. 
Theorem 4.8. (Structure Theorem for reductive Lie superalgebras) Let $\mathfrak{g}$ be a reductive Lie superalgebra generated by $\mathfrak{g}_{\overline{1}}$ with $\mathfrak{z}(\mathfrak{g}) \subseteq \mathfrak{g}_{\overline{0}}$. Then $\mathfrak{g} \cong[\mathfrak{g}, \mathfrak{g}]+\mathfrak{b}$ is a direct sum of vector spaces and the commutator algebra can be written as a sum of ideals of $\mathfrak{g}$ as follows:

$$
[\mathfrak{g}, \mathfrak{g}]=\mathfrak{z}(\mathfrak{g})+\sum_{j \in J_{s}} \mathfrak{g}(j)+\sum_{k \in J_{a}} \mathfrak{c}(k) .
$$

Moreover, the summation map

$$
S: \bigoplus_{j \in J_{s}} \mathfrak{g}(j) \oplus \bigoplus_{k \in J_{a}} \mathfrak{c}(k) \rightarrow \mathfrak{g}
$$

has central kernel.

Proof. It is clear that the ideals $\mathfrak{g}(j)$ and $\mathfrak{c}(k)$ are contained in the commutator algebra $[\mathfrak{g}, \mathfrak{g}]$ and $[\mathfrak{g}, \mathfrak{g}]_{\overline{1}}=\mathfrak{a}$, as well as

$$
[\mathfrak{g}, \mathfrak{g}]_{\overline{0}}=\mathfrak{g}_{\overline{0}}=\left[\mathfrak{g}_{\overline{1}}, \mathfrak{g}_{\overline{1}}\right]=[\mathfrak{a}, \mathfrak{a}]+[\mathfrak{a}, \mathfrak{b}]+[\mathfrak{b}, \mathfrak{b}] .
$$

As $[\mathfrak{b}, \mathfrak{b}]$ is central, $\left[\mathfrak{b}, \mathfrak{a}_{j}\right] \subseteq \mathfrak{g}(j)$ for $j \in J_{s}($ Lemma $4.3(\mathrm{i})),\left[\mathfrak{b}, \mathfrak{a}_{k}\right] \subseteq \mathfrak{c}(k)$ for $k \in J_{a}$ and

$$
[\mathfrak{a}, \mathfrak{a}] \subseteq \mathfrak{z}(\mathfrak{g})+\sum_{j \in J_{s}}\left[\mathfrak{a}_{j}, \mathfrak{a}_{j^{\prime}}\right]
$$

(Lemma 4.2 and Lemma 4.3(ii)), we thus obtain

$$
[\mathfrak{g}, \mathfrak{g}]=\mathfrak{z}(\mathfrak{g})_{\overline{0}}+\sum_{j \in J_{s}} \mathfrak{g}(j)+\sum_{k \in J_{a}} \mathfrak{c}(k) .
$$

Now let $x_{k} \in \mathfrak{c}(k)$ and $y_{j} \in \mathfrak{g}(j)$. If $j \neq j^{\prime}$, we assume that either $y_{j}$ or $y_{j^{\prime}}$ vanishes. Suppose that $z:=\sum_{k \in J_{a}} x_{k}+\sum_{j \in J_{s}} y_{j} \in \mathfrak{z}(\mathfrak{g})$. Since the sum of the subspaces $\mathfrak{a}_{j}$ and $\mathfrak{a}_{k}$ of $\mathfrak{a}$ is direct, all summands $x_{k}$ and $y_{j}$ are even and so they commute with $\mathfrak{b}$. For $k \in J_{a}$, the element $z-x_{k}$ commutes with $\mathfrak{a}_{k}$, so that $x_{k}$ does as well. As it also acts trivial on a complement of $\mathfrak{a}_{k}$ in $\mathfrak{a}$, it commutes with $\mathfrak{g}_{1}$, and thus $x_{k} \in \mathfrak{z}(\mathfrak{g})$ because $\mathfrak{g}$ is generated by $\mathfrak{g}_{\overline{1}}$. Likewise $z-y_{j}$ acts trivially on $\mathfrak{a}_{j}+\mathfrak{a}_{j^{\prime}}$, hence $y_{j}$ does likewise and with the same argument as before we obtain $y_{j} \in \mathfrak{z}(\mathfrak{g})$.

Remark 4.9. Under the assumption that $\mathfrak{g}_{\overline{0}}=\left[\mathfrak{g}_{\overline{1}}, \mathfrak{g}_{\overline{1}}\right]$, the Lie superalgebra $\mathfrak{g}$ is perfect (that is $\mathfrak{g}=[\mathfrak{g}, \mathfrak{g}]$ ) if and only if $\mathfrak{g}_{\overline{1}}=\left[\mathfrak{g}_{\overline{0}}, \mathfrak{g}_{\overline{1}}\right]=\mathfrak{a}$, i.e., if $\mathfrak{b}=\{0\}$. If this is the case, then $J=J_{s}$, so that $\mathfrak{g}$ is a central quotient of a direct sum of the ideals $\mathfrak{g}(j)$, $j \in J_{s}$.

Remark 4.10. For the even part, we obtain with Lemma 4.3

$$
[\mathfrak{g}, \mathfrak{g}]_{\overline{0}}=\mathfrak{z}(\mathfrak{g})_{\overline{0}}+\sum_{j \in J_{s}}\left[\mathfrak{a}_{j}, \mathfrak{a}_{j^{\prime}}\right]+\sum_{k \in J_{a}}\left[\mathfrak{b}, \mathfrak{a}_{k}\right]
$$

Here the ideals $\left[\mathfrak{b}, \mathfrak{a}_{k}\right]$ of $\mathfrak{g}_{\overline{0}}$ are simple and act non-trivially only on the subspace $\mathfrak{a}_{k}$ of $\mathfrak{a}$, whereas the ideals $\left[\mathfrak{a}_{j}, \mathfrak{a}_{j^{\prime}}\right]$ are reductive and act non-trivially only on the subspace $\mathfrak{a}_{j}+\mathfrak{a}_{j^{\prime}}$ of $\mathfrak{a}$. 
Lemma 4.11. Let $\mathfrak{g}$ be a reductive Lie superalgebra with $\mathfrak{g}_{\overline{0}}=\left[\mathfrak{g}_{\overline{1}}, \mathfrak{g}_{\overline{1}}\right]$. Let $\mathfrak{z}:=$ $\mathfrak{z}_{\mathfrak{b}}(\mathfrak{a})$ be the centralizer of $\mathfrak{a}$ in $\mathfrak{b}$ and $\mathfrak{b}_{r} \subseteq \mathfrak{b}$ a complementary subspace for $\mathfrak{z}$. Then $\mathfrak{g}_{r}:=[\mathfrak{g}, \mathfrak{g}]+\mathfrak{b}_{r}$ is a Lie superalgebra and $\mathfrak{g} / \mathfrak{z}(\mathfrak{g})_{\overline{0}} \cong \mathfrak{g}_{r} / \mathfrak{z}(\mathfrak{g})_{\overline{0}} \oplus \overline{\mathfrak{z}}$ is a direct sum of Lie superalgebras, where the image $\overline{\mathfrak{z}}$ of $\mathfrak{z}$ in $\mathfrak{g} / \mathfrak{z}(\mathfrak{g})_{\overline{0}}$ is a central ideal and $\mathfrak{g}_{r} / \mathfrak{z}(\mathfrak{g})_{\overline{0}}$ is semisimple.

Proof. The image $\overline{\mathfrak{z}}$ of $\mathfrak{z}$ in $\mathfrak{g} / \mathfrak{z}(\mathfrak{g})_{\overline{0}}$ is an abelian ideal because $\left[\mathfrak{z}, \mathfrak{g}_{\overline{1}}\right]=[\mathfrak{z}, \mathfrak{b}] \subseteq \mathfrak{z}(\mathfrak{g})_{\overline{0}}$ and $\left[\mathfrak{g}_{\overline{0}}, \mathfrak{z}\right]=\{0\}$. We conclude that $\mathfrak{g} / \mathfrak{z}(\mathfrak{g})_{\overline{0}}$ is a direct sum of $\mathfrak{g}_{r} / \mathfrak{z}(\mathfrak{g})_{\overline{0}}$ and $\overline{\mathfrak{z}}$.

It remains to show that $\mathfrak{g}_{r} / \mathfrak{z}(\mathfrak{g})_{\overline{0}}$ is semisimple, i.e., that every solvable ideal $\mathfrak{r} \unlhd \mathfrak{g}_{r}$ is contained in $\mathfrak{z}(\mathfrak{g})_{\overline{0}}$. First we observe that $\mathfrak{r}_{\overline{0}}$ is a solvable ideal of the reductive Lie algebra $\mathfrak{g}_{\overline{0}}$, hence central in $\mathfrak{g}_{\overline{0}}$. Since the subspaces $\mathfrak{a}_{k}, k \in J_{a}$, and $\mathfrak{a}_{j}+\mathfrak{a}_{j^{\prime}}, j \in J_{s}$, are pairwise non-equivalent $\mathfrak{g}_{0}$-modules by Remark 4.10, the subspace $\mathfrak{r}_{\overline{1}} \subseteq \mathfrak{g}_{r, \overline{1}}$ is adapted to the decomposition $\mathfrak{g}_{r, \overline{1}}=\mathfrak{b}_{r}+\sum_{k \in J_{a}} \mathfrak{a}_{k}+\sum_{j \in J_{s}} \mathfrak{a}_{j}+\mathfrak{a}_{j^{\prime}}$. If $k \in J_{a}$ and $\mathfrak{r}_{\overline{1}} \cap \mathfrak{a}_{k} \neq\{0\}$, then $\mathfrak{a}_{k} \subseteq \mathfrak{r}$, and the relation $\left[\mathfrak{b}_{r}, \mathfrak{a}_{k}\right]=\left[\mathfrak{b}, \mathfrak{a}_{k}\right] \subseteq \mathfrak{r}$ contradicts the solvability of $\mathfrak{r}$. If $j \in J_{s}$, then $\mathfrak{r} \cap \mathfrak{g}(j)$ is a solvable ideal of $\mathfrak{g}(j)$, hence central (Proposition 4.5(iii)), so that $\mathfrak{r} \cap\left(\mathfrak{a}_{j}+\mathfrak{a}_{j^{\prime}}\right)=\{0\}$. This implies that $\mathfrak{r}_{\overline{1}} \subseteq \mathfrak{b}_{r}$. Now let $b \in \mathfrak{r}_{\overline{1}}$. If $\left[b, \mathfrak{a}_{k}\right] \neq\{0\}$ for some $k \in J_{a}$, the simplicity of the ideal $\left[b, \mathfrak{a}_{k}\right]$ of $\mathfrak{g}_{\overline{0}}$ contradicts $\mathfrak{r}_{\overline{0}} \subseteq \mathfrak{z}\left(\mathfrak{g}_{\overline{0}}\right)$. If $\left[b, \mathfrak{a}_{j}\right] \neq\{0\}$ for some $j \in J_{s}$, then $\left[b, \mathfrak{a}_{j}\right] \subseteq\left[\mathfrak{g}_{\overline{0}}, \mathfrak{g}_{\overline{0}}\right]$ (Lemma $4.3(\mathrm{i})$ ) shows that $\left[b, \mathfrak{a}_{j}\right]$ is semisimple, which also contradicts the solvability of $\mathfrak{r}$. We conclude that $\left[\mathfrak{r}_{\overline{1}}, \mathfrak{a}\right]=\{0\}$. Hence $\mathfrak{r}_{\overline{1}} \subseteq \mathfrak{z} \cap \mathfrak{b}_{r}=\{0\}$ and thus $\mathfrak{r}=\mathfrak{r}_{\overline{0}} \subseteq \mathfrak{g}_{\overline{0}}$. As $\mathfrak{r}$ is an ideal, we derive that $\left[\mathfrak{r}, \mathfrak{g}_{\overline{1}}\right] \subseteq \mathfrak{r} \cap \mathfrak{g}_{\overline{1}}=\{0\}$, and since $\mathfrak{g}_{\overline{1}}$ generates $\mathfrak{g}$, we see that $\mathfrak{r}=\mathfrak{r}_{\overline{0}}$ is central in $\mathfrak{g}$, so $\mathfrak{r} \subseteq \mathfrak{z}(\mathfrak{g})_{\overline{0}}$. We conclude that every solvable ideal in $\mathfrak{g}_{r} / \mathfrak{z}(\mathfrak{g})_{\overline{0}}$ is trivial.

Remark 4.12. Let $\Lambda_{n}(\mathbb{K})$ be the Graßmann algebra of degree $n$ over $\mathbb{K}$ and $\mathbf{W}_{n}(\mathbb{K}):=$ $\operatorname{der}\left(\Lambda_{n}(\mathbb{K})\right)$ for the Lie superalgebra of its derivations. For a semisimple Lie superalgebra $\mathfrak{s}$, a slight modification of the arguments in Ch95 shows that there exist real simple Lie superalgebras $\mathfrak{s}_{1}, \ldots, \mathfrak{s}_{k}$ and nonnegative integers $n_{1}, \ldots, n_{k}$ such that

$$
\bigoplus_{i=1}^{k}\left(\mathfrak{s}_{i} \otimes_{\mathbb{K}_{i}} \Lambda_{n_{i}}\left(\mathbb{K}_{i}\right)\right) \subseteq \mathfrak{s} \subseteq \bigoplus_{i=1}^{k}\left(\operatorname{der}_{\mathbb{K}_{i}}\left(\mathfrak{s}_{i}\right) \otimes_{\mathbb{K}_{i}} \Lambda_{n_{i}}\left(\mathbb{K}_{i}\right)+\mathbb{L}_{i} \otimes_{\mathbb{K}_{i}} \mathbf{W}_{n_{i}}\left(\mathbb{K}_{i}\right)\right)
$$

where, for $1 \leq i \leq k, \mathbb{L}_{i}$ is the centroid of $\mathfrak{s}_{i}$ and $\mathbb{K}_{i} \subseteq \mathbb{L}_{i}$ is the subfield killed by $\operatorname{der}\left(\mathfrak{s}_{i}\right)$.

If, in addition, $\mathfrak{s}$ is reductive, then the Lie algebras $\left(\mathfrak{s}_{i}\right)_{\overline{0}} \otimes \Lambda_{n_{i}}\left(\mathbb{K}_{i}\right)_{\overline{0}}$ must be reductive, which immediately implies that $n_{i} \leq 1$ (cf. [El96]). The information contained in our Structure Theorem 4.8 is much finer because we obtain

$\mathfrak{g}_{r} / \mathfrak{z}(\mathfrak{g})_{\overline{0}}=\mathfrak{g}_{r} / \mathfrak{z}\left(\mathfrak{g}_{r}\right) \cong \bigoplus_{j \in J_{s}} \mathfrak{g}(j) / \mathfrak{z}(\mathfrak{g}(j)) \oplus \bigoplus_{k \in J_{a}} T \mathfrak{k}_{k}=\bigoplus_{j \in J_{s}} \mathfrak{g}(j) / \mathfrak{z}(\mathfrak{g}(j)) \oplus \bigoplus_{k \in J_{a}} \mathfrak{k}_{k} \otimes \Lambda_{1}(\mathbb{K})$ where $\mathfrak{g}(j) / \mathfrak{z}(\mathfrak{g}(j))$ is simple.

\section{The structure of unitary Lie superalgebras}

We now show how the decomposition derived in the previous section can be refined to unitary Lie superalgebras. The following lemma helps us to understand, for a com- 
pact Lie superalgebra, the structure of $\mathfrak{g}(j)$ in terms of the simple adjoint quotient (Proposition 4.5).

Lemma 5.1. Let $\mathfrak{k}$ be a compact simple Lie superalgebra and $\widehat{\mathfrak{k}}$ be a central extension with $\mathfrak{z}(\widehat{\mathfrak{k}}) \subseteq[\widehat{\mathfrak{k}}, \widehat{\mathfrak{k}}]$. Then $\operatorname{dim} \mathfrak{z}(\widehat{\mathfrak{k}}) \leq 1$.

More specifically, for $\mathfrak{k}=\mathfrak{s u}(n \mid m ; \mathbb{C}), n \neq m$, and $\mathfrak{c}(n)$, all central extensions are trivial, for $\mathfrak{k}=\mathfrak{p s u}(n \mid n ; \mathbb{C})$, the Lie superalgebra $\mathfrak{s u}(n \mid n ; \mathbb{C})$ is the universal central extension, and $\mathfrak{q}(n)$ is the universal central extension of $\mathfrak{p q}(n)$.

Proof. Write $\mathfrak{z}:=\mathfrak{z}(\widehat{\mathfrak{k}})=\mathfrak{z} \overline{0} \oplus \mathfrak{z} \overline{1}$. Then $\mathfrak{z}$ acts trivially on $\widehat{\mathfrak{k}}$, so that the adjoint action of $\widehat{\mathfrak{k}}_{\overline{0}}$ factors through an action of $\mathfrak{k}_{\overline{0}}$. Our assumption that $\mathfrak{z}(\widehat{\mathfrak{k}})$ is contained in $\left.\widehat{\mathfrak{k}}, \widehat{\mathfrak{k}}\right]$ implies that $\widehat{\mathfrak{k}}=[\widehat{\mathfrak{k}}, \widehat{\mathfrak{k}}]$, so that the bracket map of $\widehat{\mathfrak{k}}$ factors through a surjective linear map

$$
\mathfrak{k} \otimes \mathfrak{k} \rightarrow \widehat{\mathfrak{k}}
$$

Since $\mathfrak{k}$ is a semisimple $\mathfrak{k}_{\overline{0}}$-module, so is $\mathfrak{k} \otimes \mathfrak{k}$, and therefore $\widehat{\mathfrak{k}}$ also is a semisimple $\mathfrak{k}_{\overline{0}}$-module. We conclude that there exists an $\mathfrak{k}_{\overline{0}}$-invariant complement of the center $\mathfrak{z}$. We may thus write

$$
\widehat{\mathfrak{k}}=\mathfrak{z} \oplus_{\omega} \mathfrak{k} \quad \text { with } \quad\left[(z, x),\left(z^{\prime}, x^{\prime}\right)\right]=\left(\omega\left(x, x^{\prime}\right),\left[x, x^{\prime}\right]\right),
$$

where $\omega: \mathfrak{k} \times \mathfrak{k} \rightarrow \mathfrak{z}$ is a 2-cocycle. Since the corresponding decomposition of $\widehat{\mathfrak{k}}$ is $\mathfrak{k}_{\overline{0}}$-invariant, $\omega\left(\mathfrak{k}_{\overline{0}}, \mathfrak{k}\right)=\{0\}$. Therefore $\omega$ is of the form

$$
\omega\left(x_{0}+x_{1}, y_{0}+y_{1}\right)=\beta\left(x_{1}, y_{1}\right),
$$

where $\beta: \mathfrak{k}_{\overline{1}} \times \mathfrak{k}_{\overline{1}} \rightarrow \mathfrak{z} \overline{0}$ is an $\mathfrak{k}_{\overline{0}}$-invariant symmetric bilinear map. In particular, the odd component $\beta_{\overline{1}}$ vanishes.

Since the $\mathfrak{k}_{\overline{0}}$-module $\mathfrak{k}_{\overline{1}}$ is simple, the space $S^{2}\left(\mathfrak{k}_{\overline{1}}\right)^{\mathfrak{k}_{\overline{0}}}$ is one-dimensional. In fact, if $\langle\cdot, \cdot\rangle$ is an $\mathfrak{k}_{\overline{0}}$-invariant positive definite scalar product on $\mathfrak{k}_{\overline{1}}$, then any other invariant symmetric bilinear form $\gamma$ can be written as $\gamma(x, y)=\langle D x, y\rangle$ for a symmetric $\mathfrak{k}_{\overline{0}^{-}}$ intertwining operator $D$. Then the diagonalizability of $D$ implies that $D \in \mathbb{R} \mathbf{1}$, so that $\gamma$ is a multiple of the scalar product, and thus $S^{2}\left(\mathfrak{k}_{\overline{1}}\right)^{\mathfrak{k}_{\overline{0}}}$ is one-dimensional. Now the perfectness of $\widehat{\mathfrak{k}}$ implies that $\operatorname{dim} \mathfrak{z} \leq 1$.

For $\mathfrak{k} \cong \mathfrak{s u}(n \mid m ; \mathbb{C}), n \neq m$, and $\mathfrak{c}(n)$, all central extensions are trivial because their complexification has this property by Remark 2.1(c). In view of $\operatorname{dim} \mathfrak{z} \leq 1, \mathfrak{s u}(n \mid n ; \mathbb{C})$ must be the universal central extension of $\mathfrak{p s u}(n \mid n ; \mathbb{C})$, and likewise $\mathfrak{q}(n)$ is the universal central extension of $\mathfrak{p q}(n)$.

Theorem 5.2. If $\mathfrak{g}$ is unitary and $j \in J_{s}$, then the ideal $\mathfrak{g}(j)$ is isomorphic to one of the following Lie superalgebras

$$
\mathfrak{s u}(n \mid m ; \mathbb{C}), n \geq m \geq 1, \quad \mathfrak{q}(n), n \geq 2, \quad \text { or } \quad \mathfrak{c}(n), n \geq 2 .
$$

In addition, we have:

(a) If $\mathfrak{g}(j) \cong \mathfrak{s u}(n \mid m ; \mathbb{C}), n \neq m$, or $\mathfrak{g}(j) \cong \mathfrak{c}(n)$, then it is a direct summand of $\mathfrak{g}$. 
(b) If $\mathfrak{g}(j) \cong \mathfrak{s u}(n \mid n ; \mathbb{C})$, then $[\mathfrak{b}, \mathfrak{g}(j)]=\{0\}$.

(c) If $\mathfrak{g}(j) \cong \mathfrak{q}(n)$ and $\left[\mathfrak{b}, \mathfrak{a}_{j}\right] \neq\{0\}$, then we obtain an embedding $\widehat{\mathfrak{q}}(n) \hookrightarrow \mathfrak{g}$.

Proof. We consider the compact simple Lie superalgebra $\mathfrak{k}:=\mathfrak{g}(j) / \mathfrak{z}(\mathfrak{g}(j)$ ) (Proposition 4.5(iii)). In view of Theorem 2.3 it is isomorphic to one of the following

$$
\mathfrak{s u}(n \mid m ; \mathbb{C}), n>m \geq 1, \quad \mathfrak{p} \mathfrak{s u}(n \mid n ; \mathbb{C}), \quad \mathfrak{p q}(n), n \geq 2, \quad \text { or } \quad \mathfrak{c}(n), n \geq 2 .
$$

For $\mathfrak{s u}(n \mid m ; \mathbb{C}), n>m$, and $\mathfrak{c}(n)$, all central extensions are trivial by Lemma 5.1. Since $\mathfrak{g}(j)$ is perfect, we obtain in this case $\mathfrak{k}=\mathfrak{g}(j)$.

Lemma 5.1 also implies that the simple Lie superalgebras $\mathfrak{p s u}(n \mid n ; \mathbb{C})$ and $\mathfrak{p q}(n)$ have a unique non-trivial central extension by a one-dimensional center. As $\mathfrak{z}\left(\mathfrak{k}_{\overline{0}}\right)=\{0\}$ in this case, the Lie superalgebra $\mathfrak{k}$ is not unitary. Therefore $\mathfrak{g}(j)$ must be isomorphic to the unique central extension $\widehat{\mathfrak{k}}$.

(a) Let $\mathfrak{k}$ be a simple compact Lie superalgebra. If $\mathfrak{k} \cong \mathfrak{s u}(n \mid m ; \mathbb{C}), n \neq m$, or $\mathfrak{k} \cong \mathfrak{c}(n)$, then all derivations of $\mathfrak{k}$ are inner by Remark 2.1(c). This implies that, in any Lie superalgebra $\mathfrak{g}$, an ideal isomorphic to $\mathfrak{k}$ is a direct summand because $\mathfrak{g} \cong \mathfrak{k} \oplus \mathfrak{z}_{\mathfrak{g}}(\mathfrak{k})$.

(b) If $\mathfrak{k} \cong \mathfrak{p s u}(n \mid n ; \mathbb{C})$ or $\mathfrak{p q}(n)$, then the situation is more complicated. From Lemma 5.1 we know that $\mathfrak{k}$ has a universal central extension isomorphic to $\mathfrak{s u}(n \mid n ; \mathbb{C})$ or $\mathfrak{q}(n)$, respectively. In particular, all derivations of $\mathfrak{k}$ lift in a unique fashion to derivations of $\widehat{\mathfrak{k}}$, so that $\operatorname{der}(\widehat{\mathfrak{k}}) \cong \operatorname{der}(\mathfrak{k})$.

In $\operatorname{der}(\mathfrak{k})$ the subspace adk $\cong \mathfrak{k}$ of inner derivations is an ideal, and adk $\mathfrak{k}_{\overline{0}}$ is compactly embedded in $\operatorname{der}(\mathfrak{k})$. We therefore have an $\mathfrak{k}_{\overline{0}}$-invariant splitting

$$
\operatorname{der}(\mathfrak{k})_{\overline{1}} \cong \operatorname{ad}\left(\mathfrak{k}_{\overline{1}}\right) \oplus \mathfrak{d}_{\overline{1}},
$$

where all derivations in $\mathfrak{d}_{\overline{1}}$ commute with $\mathfrak{k}_{\overline{0}}$, i.e., $\mathfrak{k}_{\overline{0}} \subseteq \operatorname{ker} D$ for $D \in \mathfrak{d}_{\overline{1}}$. Since any such $D$ is odd, this implies that $D^{2}=0$.

Any $D \in \operatorname{der}(\mathfrak{k})_{\overline{1}}^{\mathfrak{k}_{\overline{0}}}$ yields an $\mathfrak{k}_{\overline{0}}$-intertwining operator $\mathfrak{k}_{\overline{1}} \rightarrow \mathfrak{k}_{\overline{0}}$. Conversely, an $\mathfrak{k}_{\overline{0}}$ intertwining operator $D: \mathfrak{k}_{\overline{1}} \rightarrow \mathfrak{k}_{\overline{0}}$ extends to an odd derivation annihilating $\mathfrak{k}_{\overline{0}}$ if and only if

$$
[D x, y]=[x, D y] \quad \text { for } \quad x, y \in \mathfrak{k}_{\overline{1}} .
$$

For $\mathfrak{k} \cong \mathfrak{p s u}(n \mid n ; \mathbb{C})$, the space $\mathfrak{k}_{\overline{1}} \cong M_{n}(\mathbb{C}) \cong \mathbb{C}^{n} \otimes \overline{\mathbb{C}^{n}}$ is a simple $\mathfrak{k}_{\overline{0}}$-module not isomorphic to any of the two ideals of $\mathfrak{k}_{\overline{0}}$. Therefore $\operatorname{Hom}_{\mathfrak{k}_{\overline{0}}}\left(\mathfrak{k}_{\overline{1}}, \mathfrak{k}_{\overline{0}}\right)=\{0\}$ implies that $\mathfrak{d}_{\overline{1}}=\{0\}$. Accordingly, $[\mathfrak{b}, \mathfrak{g}(j)]=\{0\}$ for any ideal of this type.

(c) For $\mathfrak{k} \cong \mathfrak{p q}(n)$, we have $\mathfrak{k}_{\overline{0}} \cong \mathfrak{s u}(n+1, \mathbb{C})$ and $\mathfrak{k}_{\overline{1}} \cong \mathfrak{s u}(n+1, \mathbb{C})$ with respect to the adjoint action. As $\mathfrak{s u}(n+1, \mathbb{C})_{\mathbb{C}} \cong \mathfrak{s l}(n+1, \mathbb{C})$ is a simple complex Lie algebra, the $\mathfrak{k}_{\overline{0}}$-module $\mathfrak{k}_{\overline{1}}$ is absolutely simple, i.e., $\operatorname{End}_{\mathfrak{k}_{\overline{0}}}\left(\mathfrak{k}_{\overline{1}}\right)=\mathbb{R} \mathbf{1}$. This implies that $\operatorname{dim} \mathfrak{d}_{\overline{1}} \leq 1$. We have already seen in Example 2.2(c) that a non-zero odd outer derivation exists. For an ideal of this type, a one-dimensional quotient of $\mathfrak{b}$ may act non-trivially on $\mathfrak{g}(j)$. For $\mathfrak{g}(j) \cong \mathfrak{q}(n)$ the relation $\left[\mathfrak{b}, \mathfrak{a}_{j}\right] \neq\{0\}$ therefore leads to an embedding of $\widehat{\mathfrak{q}}(n) \hookrightarrow \mathfrak{g}$ (cf. Example 2.2(c)). 
Example 5.3. Let $\mathfrak{k}$ be a simple compact Lie algebra and $T \mathfrak{k}=\mathfrak{k} \otimes \Lambda_{1}$, be as in Example 4.6. We write $\widetilde{T} \mathfrak{k}$ for the central extension of $T \mathfrak{k}$ corresponding to a positive definite symmetric bilinear form $\kappa$ on $\mathfrak{k} \cong \mathfrak{k} \otimes \xi$ and $\mathfrak{z} \cong \mathbb{R}$ for the corresponding center. Then $\mathfrak{h}:=\mathfrak{z}+(\mathfrak{k} \otimes \xi)$ is a Clifford-Heisenberg Lie superalgebra and $\widetilde{T} \mathfrak{k} \cong \mathfrak{h} \rtimes \mathfrak{k}$. Now the faithful spin representation of $\mathfrak{h}$ (cf. Subsection 3.1) also carries a natural representation of $\mathfrak{k}$ because $\kappa$ is $\mathfrak{k}$-invariant. Therefore $\widetilde{T} \mathfrak{k}$ is a unitary Lie superalgebra.

If $\mathfrak{h} \rightarrow T \mathfrak{k}$ is a non-trivial central extension of Lie superalgebras with $\mathfrak{z}(\mathfrak{h}) \subseteq[\mathfrak{h}, \mathfrak{h}]$, then the same arguments as in the proof of Lemma 5.1 imply that $\mathfrak{h} \cong \widetilde{T} \mathfrak{k}$, so that $\widetilde{T} \mathfrak{k}$ is the universal central extension of $T \mathfrak{k}$. Since $T \mathfrak{k}$ is not unitary, it follows that, if $\mathfrak{g}$ is unitary and $k \in J_{a}$, then the ideal $\mathfrak{c}(k) \unlhd \mathfrak{g}$ is isomorphic to $\widetilde{T} \mathfrak{k}_{k}$.

We conclude this paper with the following theorem which asserts that all possible types of subalgebras $\mathfrak{g}(j)$ and $\mathfrak{c}(k)$ actually occur in unitary Lie superalgebras.

Theorem 5.4. The Lie superalgebras

$$
\mathfrak{s u}(n \mid m ; \mathbb{C}), n \geq m \geq 1, \quad \mathfrak{q}(n), n \geq 2, \quad \text { or } \quad \mathfrak{c}(n), n \geq 2,
$$

and the Lie superalgebras $\widetilde{T} \mathfrak{k}, \mathfrak{k}$ compact simple, are unitary.

Proof. For $\widetilde{T} \mathfrak{k}$, the unitarity was shown in Example 5.3 , and for $\mathfrak{s u}(n \mid m ; \mathbb{C})$ and $\mathfrak{q}(n)$, the unitarity follows from the definition. Therefore it remains to show that $\mathfrak{c}(n)$ is unitary.

To this end we use Jakobsen's classification of unitary highest weight modules of basic classical Lie superalgebras. In terms of [Ja94, Ch. 9] the antilinear antiinvolution

$\omega: \mathfrak{c}(n)_{\mathbb{C}} \rightarrow \mathfrak{c}(n)_{\mathbb{C}}, \quad x_{0}+x_{1}+i\left(y_{0}+y_{1}\right) \mapsto-x_{0}-i x_{1}-i\left(-y_{0}-i y_{1}\right)=-x_{0}-y_{1}+i\left(-x_{1}+y_{0}\right)$

corresponds to the signs

$$
\varepsilon_{z}=1=\varepsilon_{p_{1}}=\varepsilon_{p_{2}} .
$$

For the corresponding real form, Ja94, Props. 9.7,9.8] represents a classification of the corresponding unitary highest weight modules. Since these modules are locally finite for the subalgebra $\mathfrak{g}_{\overline{0}}$ whose real form is compact, Ka77, Prop. 5.2.5] implies that they are finite dimensional. We conclude that the real Lie superalgebra $\mathfrak{c}(n)$ is unitary.

Acknowledgements. S. Azam acknowledges the support of IPM (Grant No. 93160221), and the Banach Algebra Center of Excellence for Mathematics. He would like to thank the people of Department Mathematik, Friedrich-Alexander Universität, for their hospitality during his visit.

K.-H. Neeb acknowledges the support of DFG-grant NE 413/7-2 in the framework of the SPP "Representation Theory".

\section{References}

[Be00] S. Benayadi, Quadratic Lie superalgebras with the completely reducible action of the even part on the odd part, J. Algebra 223 (2000), 344-366. 
[BR78] F. A. Berezin, V. Retakh, The structure of Lie superalgebras with semisimple even part, Funct. Anal. Appl. 12:1 (1978),48-50.

[CCTV06] C. Carmeli, G. Cassinelli, A. Toigo, V. S. Varadarajan, Unitary representations of super Lie groups and applications to the classification and multiplet structure of super particles, Comm. Math. Phys. 263:1 (2006), 217-258.

[Ch95] S.-J. Cheng, Differentiably simple Lie superalgebras and representations of semisimple Lie superalgebras, J. Algebra 173:1 (1995), 1-43.

[E196] A. Elduque, Lie superalgebras with semisimple even part, J. Algebra 183 (1996), 649-663.

[F13] R. Fioresi, Compact forms of complex Lie superalgebras, Preprint, arXiv:math. $\mathrm{RT}: 1205.5705 \mathrm{v} 1$.

[FG12] R. Fioresi, F. Gavarini, Chevalley supergroups, Mem. Amer. Math. Soc. 215, Amer. Math. Soc., 2012.

[HN12] J. Hilgert, K.-H. Neeb, Structure and Geometry of Lie Groups, Springer Monographs in Math., Springer, 2012.

[Ja94] H. P. Jakobsen, The full set of unitarizable highest weight modules of basic classical Lie superalgebras, Mem. Amer. Math. Soc. 111 (1994), no. 532, vi+116 pp.

[Ka77] V. G. Kac, Lie superalgebras, Advances in Math. 26:1 (1977), 8-96.

[NS11] K.-H. Neeb, H. Salmasian, Lie supergroups, unitary representations, and invariant cones, in "Supersymmetry in Mathematics and Physics", R. Fioresi, S. Ferrara and V. S. Varadarajan, Eds., Lect. Notes in Math. 2027 (2011), 195-239.

[Ot95] G. T. Ottesen, Infinite Dimensional Groups and Algebras in Quantum Physics, Springer-Verlag, Lecture Notes in Physics m 27, 1995.

[Pa80] M. Parker, Classification of real simple Lie superalgebras of classical type, J. Math. Phys. 21:4 (1980), 689-697.

[Va04] Varadarajan, V. S. Supersymmetry for Mathematicians: An introduction, Courant Lecture Notes in Mathematics, 11. New York University, Courant Institute of Mathematical Sciences, New York; American Mathematical Society, Providence, RI, 2004. 